\title{
A Novel One-pot Three-step Synthesis of 2-(1-Benzofuran-2-yl)quinoline- 3-carboxylic Acid Derivatives
}

\author{
Wentao Gao, * Chaohua Zhang and Yang Li \\ Institute of Superfine Chemicals, Bohai University, Keji Street, Jinzhou, 121000, P. R. China
}

\begin{abstract}
Neste trabalho é descrito um protocolo eficiente e simples para a síntese de ácidos 2-(1-benzofurano-2-il)quinolina-3-carboxílicos, envolvendo três etapas realizadas em um único pote: síntese de éter de Williamson, hidrólise do grupo éster na posição 2 da quinolina e ciclização por condensação intramolecular entre o aldeído e o metileno na posição 2 da quinolina.
\end{abstract}

A facile and efficient one-pot three-step procedure for the preparation of 2-(1-benzofuran2-yl)quinoline-3-carboxylic acid derivatives is described, featuring three different synthetic transformations, namely Williamson ether synthesis, hydrolysis of an ester group at the quinoline ring C-3 position, and intramolecular electrophilic cyclization reaction between the aldehyde group of salicylaldehyde and the methylene at the quinoline ring C-2 position.

Keywords: one-pot, three-step, benzofuran, quinoline

\section{Introduction}

In nature's collection of biologically active heterocycles, benzo[b]furan derivatives constitute a major group..$^{1-3}$ Especially, benzo[b]furan ring systems bearing various substituents at the $\mathrm{C}-2$ position are widely distributed in nature and have been reported to have antiviral, antioxidant and antifungal activities. ${ }^{4,5}$ Since the pioneering work of Foster et al. ${ }^{6}$ on the study of benzo[b]furan at the 2-position, there is an ever increasing number of synthetic approaches towards the construction of the 2-substituted benzo[$[b]$ furan compounds. Among these, the most widely used approach for the synthesis of 2-substituted benzo[b] furans involves the palladium-catalyzed heteroannulation of 2-halophenols with a terminal alkyne via a tandem Sonogashira coupling-5-endo-dig-cyclization, largely based on the methods of Larock and his co-workers. ${ }^{7-10}$ Recently, other less popular approaches for the synthesis of 2-substituted benzo[b]furans include $p$-toluenesulfonic acid-mediated cyclization of $o$-(1-alkynyl)anisoles to obtain 2-arylsubstituted benzofurans, ${ }^{11}$ rearrangement and cyclization reactions of 2-hydroxybenzophenones with Corey-Chaykovsky reagent, ${ }^{12}$ cyclization of 2-acyloxy-1bromomethylarenes with $\mathrm{Cr}(\mathrm{II}) \mathrm{Cl}_{2} / \mathrm{BF}_{3}-\mathrm{OEt}_{2}$ catalyt, ${ }^{13}$ boron

*e-mail: bhuzh@163.com tribromide-promoted tandem deprotection-cyclization of 2-methoxyphenylacetones,,$^{14}$ (2-methoxyphenyl) methanols, ${ }^{15}$ and 2-hydroxy-3-arylpropenoic acids ${ }^{16}$ leading respectively to 2-methyl, 2-carboxy, and 2-arylbenzo[b] furans. However, these methods often require expensive catalysts and/or multi-step synthesis.

On the other hand, the quinoline ring system is an essential structural fragment of a large number of natural and unnatural compounds displaying interesting biological activities such as antimalarial, antibacterial, anti-asthmatic, antihypertensive, and anti-inflammatory. ${ }^{17-20}$ Quinolines and their derivatives have been found applications as pharmaceuticals and agrochemicals, as well as being general synthetic building blocks. ${ }^{21-24}$ For example, chimanine alkaloids, simple 2-substituted quinolines, isolated from the bark of Galipea longiflora trees of the Rutaceae family, are effective against the parasites Leishmania sp., which are the reagents of leishmaniasis, a protozoan disease of the tropical areas in South America. On the basis of the results obtained in studies with 2-substituted quinolines, the World Health Organization (WHO) has established as a priority their ability to provide oral treatment for cutaneous leishmaniasis..$^{25-27}$ Therefore, significant effort continues to be directed toward the development of new 2-substituted quinoline structures and new methods for their construction. ${ }^{28-33}$ Recently, there is much current interest in 
assembling quinoline ring with heterocyclic systems, which represent privileged moieties in medicinal chemistry, and are ubiquitous sub-structures associated with biologically active natural products. ${ }^{34-38}$ For example, 2-(furan-2-yl) quinoline-4-carboxylic acid as well as analogues (Figure 1) was reported to have the inhibition of C. albicans prolyltRNA synthetase and showed potent in vitro antifungal activities against dermatophytes. ${ }^{39,40}$
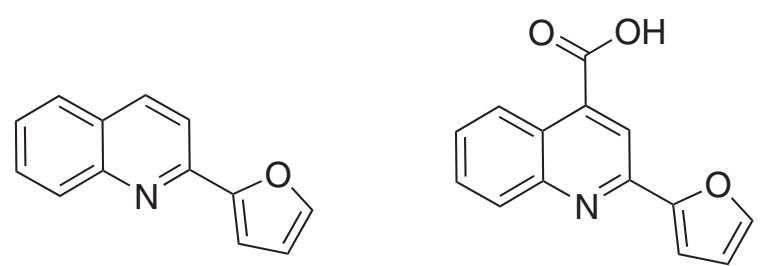

Figure 1. Structure of 2-furanylquinoline and 2-furanylquinoline-4carboxylic acid.

In light of the above reports and because of the potent biological activities of quinoline ring system and 2 -substituted benzo[b]furan derivatives, we felt that there is a real need for the synthesis of new prototypes by combining both quinoline ring system and benzo[b] furan moiety in the same molecule at 2-position, which might be important for pharmacological studies or creating new medicinal properties. Therefore, we would like to report herein a facile and inexpensive one-pot three-step procedure for the preparation of novel hybrid molecules 2-(1-benzofuran-2-yl)quinoline-3-carboxylic acid derivatives under mild conditions.

\section{Results and Discussion}

In Scheme 1 is outlined the synthetic route developed in our laboratory. The starting material ethyl 2-(bromomethyl) quinoline-3-carboxylate (1) was prepared according to the method of literature. ${ }^{41}$ The one-pot three-step reaction reported here represents a new synthetic method for the preparation of novel 2-(1-benzofuran-2-yl)quinoline3 -carboxylic acid derivatives (3a-i), involving the in situ formation of ether by Williamson reaction between compound $\mathbf{1}$ and various substituted salicylaldehydes $\mathbf{2 a - i}$ followed by the hydrolysis and intramolecular cyclization reactions. This novel procedure provides quick and easy access to the incorporation of benzo $[b]$ furan core to the quinoline nucleus at 2-position, with good yields and simple workup.

Recently, we have reported the one-pot two-step reaction of bromomethylquinolines with substituted phenols or naphthols to give the corresponding quinoline carboxylic acid ethers as key precursors of heterocycle-fused quinoline compounds. ${ }^{42,43}$ In order to continue our interest in the development of bioactive quinoline alkaloids, our initial intention was to extend the method to the reaction of monobromide 1 with salicylaldehydes, in which the presence of aldehyde functionality may provide opportunity for further manipulation. Thus, monobromide $\mathbf{1}$ was first subjected to the Williamson reaction with salicylaldehyde (2a) to give the corresponding ethyl 2-[(2-formylphenoxy)methyl] quinoline-3-carboxylate (4), which structure has already been characterized by spectroscopic method. Because the resulting $\mathbf{4}$ does not interfere with further hydrolysis reaction, purification at this stage is unnecessary. Thus, we simply added $10 \%$ ethanolic potassium hydroxide solution $10 \mathrm{~mL}$ to this reaction mixture under reflux. After the reaction was complete, it was found that the major product was identified as 2-(1-benzofuran-2-yl)quinoline-3-carboxylic acid (3a). In its ${ }^{1} \mathrm{H}$ NMR spectrum the presence of a distinct singlet, integrating for one proton resonating at $\delta 7.56 \mathrm{ppm}$, assignable to be the proton attached on the furan moiety and the absence of both the exocyclic methylene protons and the aldehyde proton resonances in product $\mathbf{3 a}$ indicated the possibility of cyclization through intramolecular condensation along with the elimination of $\mathrm{H}_{2} \mathrm{O}$. Moreover, the proposed structure of $\mathbf{3} \mathbf{a}$ was also confirmed by its ${ }^{13} \mathrm{C}$ NMR spectrum, which revealed besides the signals due to aromatic carbons the presence of carboxyl carbon at 168.16 ppm and the absence of the exocyclic methylene carbon as well as the aldehyde carbon. The IR spectrum also showed the absence of aldehyde group and exocyclic methylene<smiles></smiles> 
absorption bands. Further, the structure assigned for this reaction product was fully supported by its mass spectrum, which showed molecular ion $\left(\mathbf{M}^{+}\right)$peak at $289 \mathrm{~m} / \mathrm{z}$ and matched the expected molecular weight of 3a. Moreover, the obtained elemental analysis values are also in agreement with theoretical data.

The formation of 3a may be interpreted by the following proposed mechanism as shown in Scheme 2. The in situ base-mediated ester hydrolysis of the initially formed $\mathbf{4}$ resulted in the abstraction of acidic proton of the active methylene within the molecule $\mathbf{6}$ and subsequent attack of the carbanion at aldehydic carbon to form a five-membered cyclic system 8. After this cyclization, dehydration led to the formation of a benzofuran ring 3a. The proposed mechanism was similar to that of the formation of naphthofurans via intramolecular condensation under the action of triethyl amine reported by Srivastava et al. ${ }^{44}$

It is noteworthy that the use of $10 \%$ ethanolic potassium hydroxide solution is sufficient to promote the reaction and there were no improvements in the reaction rates and yields by increasing the amount of $\mathrm{KOH}$ or using other bases.

Encouraged by the experimental result, we attempted the reaction of monobromide $\mathbf{1}$ with a series of other salicylaldehydes (2b-i) with differing electronic properties under the similar conditions, furnishing the respective 2-benzofuranyl-quinoline-3-carboxylic acids (3b-i) in good yields as shown in Table 1.

In Table 1, a series of salicylaldehydes 2a-i (entries 1-9) reacted with 1 to give the corresponding 2-benzofuranylquinoline-3-carboxylic acids (3a-i) in 55-75\% yields. It seems that the electronic nature of the substituents has no significant effect on the reactions; all the salicylaldehydes with electron-donating (entries 2 and 3), electronwithdrawing (entries 4-6), or the halo- and $t$-butyldisubstituted groups (entries 7-9) worked well and gave the corresponding 2-benzofuranyl-quinoline-3-carboxylic acids in good yields, showing little distinction. The ease of isolation of all the products was notable; after acidification workup with $1 \mathrm{~mol} \mathrm{~L}^{-1} \mathrm{HCl}$, the products were isolated as the main products.

Additionally, since the naphthofuran backbone is a part of some natural compounds such as maturin, maturin acetate and 14-methoxydehydrocacalohastine, and novel strategies for the synthesis of naphthofuran backbone continue to receive considerable attention in the field of synthetic organic chemistry, ${ }^{45-47}$ we also attempted the reaction of $\mathbf{1}$ with<smiles>CCOC(=O)c1cc2ccccc2nc1CBr</smiles>
1<smiles>O=C(O)c1cc2ccccc2nc1-c1cc2ccccc2o1</smiles>

8<smiles>CCOC(=O)c1cc2ccccc2nc1COc1ccccc1C=O</smiles>

4<smiles>O=C([O-])c1cc2ccccc2nc1OCc1ccccc1OCCO</smiles>

7<smiles>CCOC(=O)c1cc2ccccc2nc1COc1ccccc1C=O</smiles>

5<smiles>O=Cc1ccccc1OC(CO)c1nc2ccccc2cc1C(=O)[O-]</smiles>

6

Scheme 2. Possible mechanistic pathway of formation of 2-(1-benzofuran-2-yl)quinoline-3-carboxylic acid (3a). 
Table 1. Synthesis of 2-benzofuranyl-quinoline-3-carboxylic acids (3a-i) ${ }^{\mathrm{a}}$

\begin{tabular}{|c|c|c|c|c|c|c|}
\hline Entry & Salicylaldehyde & $\mathrm{R}^{1}$ & $\mathrm{R}^{2}$ & Product & Yield / $(\%)^{\mathrm{b}}$ & $\mathrm{mp} /{ }^{\circ} \mathrm{C}$ \\
\hline 1 & $2 a$ & $\mathrm{H}$ & $\mathrm{H}$ & $3 a$ & 58 & $245.5-245.6$ \\
\hline 2 & $2 \mathbf{b}$ & $\mathrm{H}$ & $\mathrm{Me}$ & $3 \mathbf{b}$ & 57 & $240.3-241.0$ \\
\hline 3 & $2 c$ & $\mathrm{H}$ & $t-\mathrm{Bu}$ & $3 c$ & 64 & $229.2-229.6$ \\
\hline 4 & $2 d$ & $\mathrm{H}$ & $\mathrm{Cl}$ & 3d & 67 & $244.8-245.0$ \\
\hline 5 & $2 e$ & $\mathrm{H}$ & $\mathrm{Br}$ & $3 e$ & 65 & $244.0-244.2$ \\
\hline 6 & $2 f$ & $\mathrm{H}$ & $\mathrm{NO}_{2}$ & $3 f$ & 63 & $269.9-270.0$ \\
\hline 7 & $2 \mathrm{~g}$ & $\mathrm{~F}$ & $t-\mathrm{Bu}$ & $3 g$ & 55 & $224.1-224.5$ \\
\hline 8 & $2 \mathrm{~h}$ & $\mathrm{Cl}$ & $t-\mathrm{Bu}$ & $3 \mathrm{~h}$ & 75 & 217.4-218.1 \\
\hline 9 & $2 \mathrm{i}$ & $\mathrm{Br}$ & $t-\mathrm{Bu}$ & $3 \mathbf{i}$ & 67 & 224.3-225.1 \\
\hline
\end{tabular}

${ }^{\mathrm{a}} \mathrm{All}$ reactions were performed at $1 \mathrm{mmol}$ scale in $12 \mathrm{~mL}$ of $\mathrm{CH}_{3} \mathrm{CN}$. ${ }^{\text {b }}$ Yield of isolated product.<smiles>CCOC(=O)c1cc2ccccc2nc1CBr</smiles>

1<smiles>O=Cc1c(O)ccc2ccccc12</smiles>

1) $\mathrm{K}_{2} \mathrm{CO}_{3}, \mathrm{CH}_{3} \mathrm{CN}$, reflux

2) $10 \% \mathrm{KOH}$

3) $1 \mathrm{~mol} \mathrm{~L}^{-1} \mathrm{HCl}$

2j<smiles>O=C(O)c1cc2ccccc2nc1-c1cc2c(ccc3ccccc32)o1</smiles>

3j

Scheme 3. Synthesis of 2-naphtho[2,1-b]furan-2-ylquinoline-3-carboxylic acid (3j).

2-hydroxy-1-naphthaldehyde (2j) (Scheme 3) under similar conditions with the aim to construct novel naphthofuran derivative. Interestingly, 2-hydroxy-1-naphthaldehyde was equally amenable to the conditions and gave the corresponding 2-naphtho[2,1-b]furan-2-ylquinoline-3carboxylic acid (3j) in a good yield of $66 \%$.

\section{Conclusions}

In summary, the present method offered a facile synthetic route to a variety of 2-benzofuranyl-quinoline-3carboxylic acids (3a-i) as well as 2-naphtho[2,1-b]furan-2yl-quinoline-3-carboxylic acid (3j), which would provide new leads in the search for future drug candidates. Readily availability of starting material, mild reaction conditions, short reaction times, experimental simplicity and satisfactory yields contribute to the usefulness of this method. Possible biological activity of the described compounds possessing the benzofuran and quinoline skeletons remains to be studied. In addition, the products represent potentially useful synthetic building blocks in medicinal chemistry.

\section{Experimental}

\section{Chemical analysis}

Melting points (uncorrected) were determined by using WRS-1B melting points apparatus and are uncorrected. ${ }^{1} \mathrm{H}$ NMR and ${ }^{13} \mathrm{C}$ NMR spectra were recorded on a Varian Inova $400 \mathrm{MHz}$ or Brucker AVANCE $600 \mathrm{MHz}$ NMR spectrometer using $\mathrm{CDCl}_{3}$ or DMSO- $d_{6}$ as the solvent. The reported chemical shifts ( $\delta$ values) are given in parts per million downfield from tetramethylsilane (TMS) as the internal standard. EI-MS (70 eV): Finningan-MAT-8430 mass spectrometer. The elemental analyses was performed for $\mathrm{C}, \mathrm{H}$, and $\mathrm{N}$ using an Elementar Vario EL-III element analyzer and found within $\pm 0.4 \%$. The progress of reactions was monitored by thin-layer chromatography (TLC) on silica gel GF254 using ethyl acetate as eluent.

The mass spectra and elemental analysis data were in agreement with the proposed structures.

General procedure for the preparation of 2-(1-benzofuran2-yl)quinoline-3-carboxylic acids $\mathbf{3 a - i}$

A mixture of ethyl 2-(bromomethyl)quinoline-3carboxylate ( $1 \mathrm{mmol}, 0.294 \mathrm{~g})$, each salicylaldehyde (1 mmol) and $\mathrm{K}_{2} \mathrm{CO}_{3}(4 \mathrm{mmol}, 0.552 \mathrm{~g})$ was stirred in refluxing $\mathrm{CH}_{3} \mathrm{CN}(12 \mathrm{~mL})$. The conversion was monitored by TLC. After completion, $10 \%$ ethanolic potassium hydroxide solution $10 \mathrm{~mL}$ was added to the reaction mixture and continued to reflux for $2 \mathrm{~h}$, cooled, and acidified with $1 \mathrm{~mol} \mathrm{~L}^{-1} \mathrm{HCl}$ solution. The resulting crude product was recrystallized from ethanol to afford the corresponding products $3 \mathbf{a}-\mathbf{i}$ in $55-75 \%$ yields. 
The melting pints and yields of all the compounds are summarized in Table 1 and the spectral and analytical data are given below.

\section{2-(1-Benzofuran-2-yl)quinoline-3-carboxylic acid (3a)}

Orange solid. ${ }^{1} \mathrm{H}$ NMR (DMSO- $\left.d_{6}, 400 \mathrm{MHz}\right): \delta 7.32-$ 7.35 (m, 1H, Ar-H), 7.41-7.44 (m, 1H, Ar-H), 7.56 (s, 1H, furan-H), 7.64 (d, 1H, J 8.0 Hz, Ar-H), 7.71-7.74 (m, 1H, Ar-H), 7.79 (d, 1H, J 6.12 Hz, Ar-H), 7.90-7.93 (m, 1H, Ar-H), 8.12-8.16 (m, 2H, Ar-H), 8.17 (s, 1H, Ar-H), 13.5 (s, $1 \mathrm{H}, \mathrm{OH}) ;{ }^{13} \mathrm{C}$ NMR (DMSO- $\left.d_{6}, 75 \mathrm{MHz}\right): \delta 107.29,111.49$, $122.15,123.52,125.72,126.14,126.33,128.05,128.17$, 128.71, 128.84, 131.91, 137.62, 145.78, 147.35, 154.20, 154.83, 168.64; EI-MS $m / z(\%)=289\left(5 \%, \mathrm{M}^{+}\right), 244(100$, $\left.[\mathrm{M}-\mathrm{COOH}]^{+}\right), 245$ (26), 288 (18); IR (KBr) $v_{\max } / \mathrm{cm}^{-1}: 3449$ (COOH), 1719 (C=O), 1618, 1590, 1560, 1491, 1443, 1420, 1337, 1349, 1234, 1142, 793, 753; Anal. Calc. for $\mathrm{C}_{18} \mathrm{H}_{11} \mathrm{NO}_{3}$ : C, 74.73; H, 3.83; N, 4.84. Found: C, 74.69; H, 3.87; N, 4.79.

2-(5-Methyl-1-benzofuran-2-yl)quinoline-3-carboxylic $\operatorname{acid}(\mathbf{3 b})$

Orange solid. ${ }^{1} \mathrm{H}$ NMR (DMSO- $\left.d_{6}, 400 \mathrm{MHz}\right): \delta 2.44$ (s, 3H, $\mathrm{CH}_{3}$ ), 7.22-7.24 (m, 1H, Ar-H), 7.49-7.53 (m, 2H, Ar-H), 7.57 (s, 1H, furan-H), 7.70-7.73 (m, 1H, Ar-H), 7.89-7.92 (m, 1H, Ar-H), 8.12-8.15 (m, 2H, Ar-H), 8.77 (s, $1 \mathrm{H}, \mathrm{Ar}-\mathrm{H}), 13.5$ (s, $1 \mathrm{H}, \mathrm{OH}) ;{ }^{13} \mathrm{C}$ NMR (DMSO- $d_{6}$, $75 \mathrm{MHz}): \delta 21.46,107.28,111.50,121.65,124.97,125.72$, 126.06, 126.32, 127.92, 128.69, 128.80, 131.85, 135.78, 137.49, 145.82, 147.36, 153.63, 155.31, 168.74; EI-MS m/z $(\%)=303\left(55 \%, \mathrm{M}^{+}\right), 258\left(100,[\mathrm{M}-\mathrm{COOH}]^{+}\right), 259(38)$, 302 (71); IR (KBr) $v_{\max } / \mathrm{cm}^{-1}: 3445(\mathrm{COOH}), 1716(\mathrm{C}=\mathrm{O})$, 1618, 1592, 1558, 1490, 1445, 1417, 1376, 1326, 1231, 1200, 793, 742; Anal. Calc. for $\mathrm{C}_{19} \mathrm{H}_{13} \mathrm{NO}_{3}$ : C, 75.24; $\mathrm{H}$, 4.32; N, 4.62. Found: C, 75.27; H, 4.39; N, 4.57.

\section{2-(5-tert-Butyl-1-benzofuran-2-yl)quinoline-3-carboxylic} $\operatorname{acid}(3 c)$

White solid. ${ }^{1} \mathrm{H}$ NMR $\left(\mathrm{CDCl}_{3}, 400 \mathrm{MHz}\right): \delta 1.38(\mathrm{~s}, 9 \mathrm{H}$, $t$-butyl), 7.36-7.38 (m, 1H, Ar-H), 7.42 (d, 1H, J 8.7 Hz, Ar-H), 7.52 (s, 1H, furan-H), 7.59-7.62 (m, 1H, Ar-H), $7.64(\mathrm{~d}, 1 \mathrm{H}, J 1.7 \mathrm{~Hz}, \mathrm{Ar}-\mathrm{H}), 7.79-7.82(\mathrm{~m}, 1 \mathrm{H}, \mathrm{Ar}-\mathrm{H})$, $7.88(\mathrm{~d}, 1 \mathrm{H}, J 8.0 \mathrm{~Hz}, \mathrm{Ar}-\mathrm{H}), 8.21$ (d, 1H, J $8.4 \mathrm{~Hz}, \mathrm{Ar}-\mathrm{H})$, 8.63 (s, $1 \mathrm{H}, \mathrm{Ar}-\mathrm{H}) ;{ }^{13} \mathrm{C} \mathrm{NMR}\left(\mathrm{CDCl}_{3}, 150 \mathrm{MHz}\right): \delta 31.80$, $34.75,108.58,110.96,118.07,123.82,124.00,125.98$, $127.83,128.12,128.32,129.21,132.09,139.19,146.33$, 146.76, 148.16, 153.66, 153.94, 171.31; EI-MS $m / z(\%)=$ $345\left(36 \%, \mathrm{M}^{+}\right), 300\left(100,[\mathrm{M}-\mathrm{COOH}]^{+}\right), 301(42), 302(7)$, 344 (94), 346 (8); IR (KBr) $v_{\max } / \mathrm{cm}^{-1}: 3441(\mathrm{COOH}), 1712$ $(\mathrm{C}=\mathrm{O}), 1619,1597,1492,1453,1411,1324,1240,1166$, 800, 752; Anal. Calc. for $\mathrm{C}_{22} \mathrm{H}_{19} \mathrm{NO}_{3}: \mathrm{C}, 76.50 ; \mathrm{H}, 5.54 ; \mathrm{N}$, 4.06. Found: C, 76.45; H, 5.58; N, 4.11.
2-(5-Chloro-1-benzofuran-2-yl)quinoline-3-carboxylic $\operatorname{acid}(3 \boldsymbol{d})$

Orange solid. ${ }^{1} \mathrm{H}$ NMR (DMSO- $\left.d_{6}, 400 \mathrm{MHz}\right): \delta 7.42-$ $7.44(\mathrm{~m}, 1 \mathrm{H}, \mathrm{Ar}-\mathrm{H}), 7.54(\mathrm{~s}, 1 \mathrm{H}$, furan- $\mathrm{H}), 7.69(\mathrm{~d}, 1 \mathrm{H}$, $J$ 8.7 Hz, Ar-H), 7.72-7.75 (m, 1H, Ar-H), 7.87 (d, 1H, $J$ 2.15 Hz, Ar-H), 7.91-7.94 (m, 1H, Ar-H), 8.13-8.17 (m, 2H, Ar-H), 8.81 (s, 1H, Ar-H), 13.6 (s, $1 \mathrm{H}, \mathrm{OH}) ;{ }^{13} \mathrm{C} \mathrm{NMR}$ (DMSO- $d_{6}, 75$ MHz): $\delta$ 106.89, 113.11, 121.50, 125.59, $126.28,127.88,128.28,128.77,128.89,129.80,132.06$, 137.92, 145.48, 147.36, 153.28, 155.75, 168.41; EI-MS m/z $(\%)=324\left(60 \%, \mathrm{M}^{+}\right), 279\left(55,[\mathrm{M}-\mathrm{COOH}]^{+}\right), 278(100)$, 323 (84); IR (KBr) $v_{\max } / \mathrm{cm}^{-1}: 3442(\mathrm{COOH}), 1719(\mathrm{C}=\mathrm{O})$, 1618, 1593, 1558, 1491, 1457, 1376, 1323, 1231, 1173, 795, 743; Anal. Calc. for $\mathrm{C}_{18} \mathrm{H}_{10} \mathrm{ClNO}_{3}: \mathrm{C}, 66.78 ; \mathrm{H}, 3.11$; N, 4.33. Found: C, 66.82; H, 3.14; N, 4.29.

2-(5-Bromo-1-benzofuran-2-yl)quinoline-3-carboxylic acid $(3 \boldsymbol{e})$

Gray solid. ${ }^{1} \mathrm{H}$ NMR (DMSO- $\left.d_{6}, 600 \mathrm{MHz}\right): \delta 7.54-$ 7.56 (complex m, 2H, Ar-H and furan-H), $7.64(\mathrm{~d}, 1 \mathrm{H}$, $J$ 8.7 Hz, Ar-H), 7.72-7.75 (m, 1H, Ar-H), 7.91-7.94 (m, $1 \mathrm{H}, \mathrm{Ar}-\mathrm{H}), 8.01$ (d, 1H, J $2.0 \mathrm{~Hz}, \mathrm{Ar}-\mathrm{H}), 8.13-8.18$ (m, 2H, Ar-H), 8.82 (s, 1H, Ar-H), 13.6 (s, 1H, OH); EI-MS m/z $(\%)=368\left(25 \%, \mathrm{M}^{+}\right), 323\left(90,[\mathrm{M}-\mathrm{COOH}]^{+}\right), 324(44)$, 367 (65); ${ }^{13} \mathrm{C}$ NMR (DMSO- $\left.d_{6}, 150 \mathrm{MHz}\right): 107.55,113.53$, $115.34,124.05,127.16,127.24,127.72,128.81,129.55$, $130.89,133.50,136.37,144.91,145.88,153.27,155.97$, 171.18; IR (KBr) $v_{\max } / \mathrm{cm}^{-1}: 3439(\mathrm{COOH}), 1714(\mathrm{C}=\mathrm{O})$, 1619, 1593, 1559, 1491, 1434, 1377, 1324, 1254, 1232, 795, 743; Anal. Calc. for $\mathrm{C}_{18} \mathrm{H}_{10} \mathrm{BrNO}_{3}$ : C, 58.72; $\mathrm{H}, 2.74$; N, 3.80. Found: C, 58.67; H, 2.79; N, 3.76.

2-(5-Nitro-1-benzofuran-2-yl)quinoline-3-carboxylic acid (3f)

Yellow solid. ${ }^{1} \mathrm{H}$ NMR (DMSO- $d_{6}, 400 \mathrm{MHz}$ ): $\delta 7.75-$ 7.78 (complex m, 2H, Ar-H and furan-H), 7.90-7.97 (m, $2 \mathrm{H}, \mathrm{Ar}-\mathrm{H}), 8.16-8.21(\mathrm{~m}, 2 \mathrm{H}, \mathrm{Ar}-\mathrm{H}), 8.28-8.31(\mathrm{~m}, 1 \mathrm{H}$, Ar-H), 8.77 (d, 1H, J $2.3 \mathrm{~Hz}$, Ar-H), 8.88 (s, 1H, Ar-H), 13.6 (s, $1 \mathrm{H}, \mathrm{OH}) ;{ }^{13} \mathrm{C} \mathrm{NMR}$ (DMSO- $d_{6}, 75 \mathrm{MHz}$ ): $\delta 108.00$, 112.42, 118.70, 121.14, 126.17, 126.45, 128.52, 128.87, 128.96, 132.22, 138.32, 144.12, 147.39, 157.44, 168.16; EI-MS $m / z(\%)=334\left(5 \%, \mathrm{M}^{+}\right), 289\left(100,[\mathrm{M}-\mathrm{COOH}]^{+}\right)$, 290 (25), 333 (27); IR (KBr) $v_{\max } / \mathrm{cm}^{-1}: 3438(\mathrm{COOH}), 1712$ $(\mathrm{C}=\mathrm{O}), 1620,1589,1555,1520,1491,1451,1377,1344$, 1266, 789, 746; Anal. Calc. for $\mathrm{C}_{18} \mathrm{H}_{10} \mathrm{~N}_{2} \mathrm{O}_{5}$ : C, 64.67; H, 3.02; N, 8.38. Found: C, 64.76; H, 3.07; N, 8.34.

2-(5-tert-Butyl-7-fluoro-1-benzofuran-2-yl)quinoline-3carboxylic acid $(\mathbf{3 g})$

White solid. ${ }^{1} \mathrm{H}$ NMR $\left(\mathrm{CDCl}_{3}, 400 \mathrm{MHz}\right): \delta 1.36$ (s, 9H, t-butyl), 7.10-7.13 (m, 1H, Ar-H), 7.41 (d, 1H, $J$ 
$1.6 \mathrm{~Hz}, \mathrm{Ar}-\mathrm{H}), 7.52$ (s, 1H, furan-H), 7.61-7.65 (m, 1H, Ar-H), 7.82-7.85 (m, 1H, Ar-H), 7.91 (d, 1H, J 8.0 Hz, Ar-H), 8.20 (d, 1H, J 8.4 Hz, Ar-H), 8.67 (s, 1H, Ar-H); ${ }^{13} \mathrm{C}$ NMR (DMSO- $d_{6}, 150 \mathrm{MHz}$ ): 31.42, 34.87, 107.79, 109.74, 109.84, 113.98, 126.26, 128.21, 128.77, 128.87, 131.36, 132.01, 137.94, 139.55, 139.63, 145.51, 145.86, $147.34,147.49,148.05,155.41,168.39 ;$ EI-MS $m / z(\%)=$ $363\left(80 \%, \mathrm{M}^{+}\right), 318\left(100,[\mathrm{M}-\mathrm{COOH}]^{+}\right), 319(30), 362$ (95); IR (KBr) $v_{\text {max }} / \mathrm{cm}^{-1}: 3442(\mathrm{COOH}), 1711(\mathrm{C}=\mathrm{O}), 1611$, 1598, 1561, 1492, 1449, 1376, 1327, 1233, 1183, 798, 750; Anal. Calc. for $\mathrm{C}_{22} \mathrm{H}_{18} \mathrm{FNO}_{3}$ : C, 72.72; H, 4.99; N, 3.85 . Found: C, 72.78; H, 5.01; N, 3.83 .

2-(5-tert-Butyl-7-chloro-1-benzofuran-2-yl)quinoline-3carboxylic acid $(\mathbf{3 h})$

Orange solid. ${ }^{1} \mathrm{H}$ NMR $\left(\mathrm{CDCl}_{3}, 600 \mathrm{MHz}\right): \delta 1.39(\mathrm{~s}, 9 \mathrm{H}$, t-butyl), 7.40 (d, 1H, $J 1.2 \mathrm{~Hz}, \mathrm{Ar}-\mathrm{H}), 7.57$ (d, 1H, $J 1.8 \mathrm{~Hz}$, Ar-H), 7.58 (s, 1H, furan-H), $7.65(\mathrm{~s}, 1 \mathrm{H}, \mathrm{Ar}-\mathrm{H}), 7.86(\mathrm{~s}, 1 \mathrm{H}$, Ar-H), 7.94 (d, 1H, J 7.8 Hz, Ar-H), 8.24 (d, 1H, J $8.4 \mathrm{~Hz}$, Ar-H), 8.68 (s, 1H, Ar-H); ${ }^{13} \mathrm{C} \mathrm{NMR}\left(\mathrm{CDCl}_{3}, 150 \mathrm{MHz}\right): \delta$ 31.70, 34.82, 108.61, 115.49, 116.19, 116.81, 123.88, 124.00, $126.19,128.10,128.36,129.21,129.63,132.23,139.17$, $146.11,147.98,149.64,170.13$; EI-MS $m / z(\%)=380(35 \%$, $\mathrm{M}^{+}$), 335 (35, [M-COOH] $]^{+}$), 213 (33), 215 (11), 334 (95), 378 (98), 379 (22); IR (KBr) $v_{\max } / \mathrm{cm}^{-1}: 3429(\mathrm{COOH}), 1702$ $(\mathrm{C}=\mathrm{O}), 1620,1580,1556,1479,1460,1394,1364,1286,1258$, 1165, 790, 752; Anal. Calc. for $\mathrm{C}_{22} \mathrm{H}_{18} \mathrm{ClNO}_{3}: \mathrm{C}, 69.57 ; \mathrm{H}$, 4.78; N, 3.69. Found: C, 69.64; H, 4.80; N, 3.65.

2-(7-Bromo-5-tert-butyl-1-benzofuran-2-yl)quinoline-3carboxylic acid (3i)

Orange solid. ${ }^{1} \mathrm{H} \mathrm{NMR}\left(\mathrm{CDCl}_{3}, 600 \mathrm{MHz}\right): \delta 1.39$ (s, $9 \mathrm{H}, t$-butyl), 7.55 (s, $1 \mathrm{H}$, furan-H), 7.61-7.65 (m, 3H, Ar-H), 7.84-7.87 (m, 1H, Ar-H), 7.93 (d, 1H, J 7.8 Hz, Ar-H), 8.25 (d, $1 \mathrm{H}, J 8.4 \mathrm{~Hz}, \mathrm{Ar}-\mathrm{H}), 8.68$ (s, $1 \mathrm{H}, \mathrm{Ar}-\mathrm{H}) ;{ }^{13} \mathrm{C} \mathrm{NMR}\left(\mathrm{CDCl}_{3}\right.$, $150 \mathrm{MHz}): \delta 31.73,34.94,103.57,108.55,117.47,124.22$, $126.18,126.66,128.04,128.35,129.26,132.13,139.01$, 146.10, 148.31, 151.04, 171.11; EI-MS $m / z(\%)=424(97 \%$, $\left.\mathrm{M}^{+}\right), 379$ (75, [M-COOH $]^{+}$), 257 (26), 378 (80), 423 (95); $\operatorname{IR}(\mathrm{KBr}) v_{\text {max }} / \mathrm{cm}^{-1}: 3415(\mathrm{COOH}), 1703(\mathrm{C}=\mathrm{O}), 1619,1578$, 1554, 1479, 1461, 1420, 1393, 1363, 1323, 1284, 1258, 1163 , 791, 751; Anal. Calc. for $\mathrm{C}_{22} \mathrm{H}_{18} \mathrm{BrNO}_{3}$ : C, 62.28; H, 4.28; N, 3.30. Found: C, 62.33; H, 4.32; N, 3.26.

Preparation of 2-naphtho[2,1-b]furan-2-ylquinoline-3carboxylic acid (3j)

A mixture of ethyl 2-(bromomethyl)quinoline-3carboxylate (1 mmol, $0.294 \mathrm{~g}$ ), 2-hydroxy-1-naphthaldehyde $\left(1 \mathrm{mmol}, 0.172 \mathrm{~g}\right.$ ) and $\mathrm{K}_{2} \mathrm{CO}_{3}(4 \mathrm{mmol}, 0.552 \mathrm{~g}$ ) was stirred in $\mathrm{CH}_{3} \mathrm{CN}(12 \mathrm{~mL})$ at reflux temperature. The conversion was monitored by TLC. After completion, 10\% ethanolic potassium hydroxide solution $10 \mathrm{~mL}$ was added to the reaction mixture and continued to reflux for $2 \mathrm{~h}$, cooled, and acidified with $1 \mathrm{~mol} \mathrm{~L}^{-1} \mathrm{HCl}$ solutuon. The resulting crude product was recrystallized from ethanol to afford the corresponding product 3j in $66 \%$ yield; red solid, mp 246.3-246.4 ${ }^{\circ} \mathrm{C}$. ${ }^{1} \mathrm{H}$ NMR (DMSO- $d_{6}, 400$ MHz): $\delta 7.57-7.60$ (m, 1H, Ar-H), 7.68-7.74 (complex m, 2H, Ar-H and furan-H), $7.84(\mathrm{~d}, 1 \mathrm{H}, J 8.9 \mathrm{~Hz}$, Ar-H), 7.92-7.96 (m, 2H, Ar-H), 8.09 (d, 1H, J 8.1 Hz, Ar-H), 8.15-8.17 (m, 2H, Ar-H), 8.26 (s, 1H, Ar-H), 8.48 (d, 1H, $J$ $8.1 \mathrm{~Hz}, \mathrm{Ar}-\mathrm{H}), 8.81$ (s, 1H, Ar-H), 13.6 (s, 1H, OH); ${ }^{13} \mathrm{C} \mathrm{NMR}$ (DMSO- $d_{6}, 75$ MHz): $\delta$ 106.81, 112.43, 123.70, 123.96, $125.21,126.07,126.23,126.80,127.01,127.53,127.89$, $128.74,130.15,131.92,137.65,145.83,147.44,152.64$, 153.81, 168.78; EI-MS $m / z(\%)=339\left(15 \%, \mathrm{M}^{+}\right), 294(100$, $\left.[\mathrm{M}-\mathrm{COOH}]^{+}\right), 295(47), 296(10), 338(57) ; \mathrm{IR}(\mathrm{KBr}) v_{\max } / \mathrm{cm}^{-1}$ : $3442(\mathrm{COOH}), 1696(\mathrm{C}=\mathrm{O}), 1620,1589,1557,1492,1452$, 1384, 1255, 1194, 796, 751; Anal. Calc. for $\mathrm{C}_{22} \mathrm{H}_{13} \mathrm{NO}_{3}$ : C, 77.87; H, 3.86; N, 4.13. Found: C, 77.85; H, 3.89; N, 4.10.

Preparation of Ethyl 2-[(2-Formylphenoxy)methyl] quinoline-3-carboxylate (4)

A mixture of ethyl 2-(bromomethyl)quinoline-3carboxylate $(1 \mathrm{mmol}, 0.294 \mathrm{~g})$, salicylaldehyde $(1 \mathrm{mmol}$, $0.122 \mathrm{~g})$ and $\mathrm{K}_{2} \mathrm{CO}_{3}(4 \mathrm{mmol}, 0.552 \mathrm{~g})$ was stirred in refluxing $\mathrm{CH}_{3} \mathrm{CN}(12 \mathrm{~mL})$. The conversion was monitored by TLC. After completion, reaction mixture was quenched with $\mathrm{H}_{2} \mathrm{O}$. The precipitated crude product was recrystallized from ethanol to afford the corresponding product 4 in $61 \%$ yield; white solid, mp 88.6-89.1 ${ }^{\circ} \mathrm{C} .{ }^{1} \mathrm{H}$ NMR $\left(\mathrm{CDCl}_{3}, 600 \mathrm{MHz}\right): \delta 1.35$ (t, $\left.3 \mathrm{H}, J 7.2 \mathrm{~Hz}, \mathrm{OCH}_{2} \underline{\mathrm{CH}}_{3}\right), 4.38\left(\mathrm{q}, 2 \mathrm{H}, J 5.6 \mathrm{~Hz}, \mathrm{OC}_{2} \mathrm{CH}_{3}\right.$ ), 5.81 (s, 2H, Ar- $\left.\mathrm{CH}_{2}-\mathrm{O}\right), 7.01-7.04$ (m, 1H, Ar-H), 7.17 (d, $1 \mathrm{H}, J$ 8.4 Hz Ar-H), 7.51-7.54 (m, 1H, Ar-H), 7.62-7.64 (m, $1 \mathrm{H}, \mathrm{Ar}-\mathrm{H}), 7.82-7.86$ (m, 2H, Ar-H), 7.92 (d, $1 \mathrm{H}, J 7.8 \mathrm{~Hz}$, Ar-H), 8.12 (d, 1H, J 8.4 Hz, Ar-H), 8.79 (s, 1H, Ar-H), 10.52 (s, $1 \mathrm{H}, \mathrm{CHO}) ;{ }^{13} \mathrm{C}$ NMR $\left(\mathrm{CDCl}_{3}, 150 \mathrm{MHz}\right): \delta 14.12,61.77$, 71.53, 113.32, 121.00, 123.63, 125.17, 126.58, 127.78, 128.08, $128.47,129.33,131.94,135.81,140.26,148.15,154.62$, 161.40, 165.96, 189.86; EI-MS $m / z(\%)=335\left(100 \%, \mathrm{M}^{+}\right)$; $\mathrm{IR}(\mathrm{KBr}) \mathrm{V}_{\max } / \mathrm{cm}^{-1}: 2966\left(\mathrm{C}-\mathrm{H}, \mathrm{CH}_{3}\right), 2899\left(\mathrm{C}-\mathrm{H}, \mathrm{CH}_{2}\right), 2852$ (C-H, CHO), $1710(\mathrm{CHO}, \mathrm{C}=\mathrm{O}), 1685\left(\mathrm{COOC}_{2} \mathrm{H}_{5}, \mathrm{C}=\mathrm{O}\right)$, 1622, 1592, 1565, 1457, 1425, 1363, 1314, 1277, 1204, 1133, 787, 751; Anal. Calc. for $\mathrm{C}_{20} \mathrm{H}_{17} \mathrm{NO}_{4}: \mathrm{C}, 71.63 ; \mathrm{H}, 5.11 ; \mathrm{N}$, 4.18. Found: C, 71.69; H, 5.04; N, 4.23.

\section{Supplementary Information}

Supplementary information is available free of charge at http://jbcs.sbq.org.br, as PDF file. 


\section{Acknowledgments}

The authors thank the Foundation of Liaoning Province Key Laboratory of Applied Chemistry (Grant No. 2008s001) for financial support.

\section{References}

1. Schneiders, G. E.; Stevenson, R.; J. Org. Chem. 1979, 44, 4710.

2. Carlsson, B.; Singh, B. N.; Temciue, M.; Nilsson, S.; Li, Y. L.; Mellin, C.; Malm, J.; J. Med. Chem. 2002, 45, 623.

3. Shirota, O.; Pathak, V.; Sekita, S.; Stake, M.; Nagashima, Y.; Hirayama, Y.; Hakamata, Y.; Haysahi, T.; J. Nat. Prod. 2003, 66, 1128.

4. Fuganti, C.; Serra, S.; Tetrahedron Lett. 1998, 39, 5609.

5. Abdel-Wahab, B. F.; Abdel-Aziz, H. A.; Ahmed, E. M.; Eur. J. Med. Chem. 2009, 44, 2632.

6. Foster, R. T.; Robertson, A.; Bushra, A.; J. Chem. Soc. 1948 , 2254.

7. Yue, D.; Yao, T.; Larock, R. C.; J. Org. Chem. 2005, 70, 10292.

8. Larock, R. C.; Yum, E. K.; Doty, M. J.; Sham, K. K. C.; J. Org. Chem. 1995, 60, 3270.

9. Yue, D.; Larock, R. C.; J. Org. Chem. 2002, 67, 1905.

10. Larock, R. C.; Harrison, L. W.; J. Am. Chem. Soc. 1984, 106, 4218.

11. Jacubert, M.; Hamze, A.; Provot, O.; Peyrat, J. F.; Brion, J. D.; Alami, M.; Tetrahedron Lett. 2009, 50, 3588.

12. Chittimalla, S. K.; Chang, T. C.; Liu, T. C.; Hsieh, H. P.; Liao, C. C.; Tetrahedron 2008, 64, 2586.

13. Ledoussal, B.; Gorgues, A.; Le Coq, A.; Tetrahedron 1987, 43, 5841.

14. Dupont, R.; Cotelle, P.; Synthesis 1999, 1651.

15. Dupont, R.; Cotelle, P.; Tetrahedron 2001, 57, 5585.

16. Dupont, R.; Cotelle, P.; Tetrahedron Lett. 2001, 42, 597.

17. Larsen, R. D.; Corley, E. G.; King, A. O.; Carrol, J. D.; Davis, P.; Verhoeven, T. R.; Reider, P. J.; Labelle, M.; Gauthier, J. Y.; Xiang, Y. B.; Zamboni, R. J.; J. Org. Chem. 1996, 61, 3398.

18. Bilker, O.; Lindo, V.; Panico, M.; Etiene, E. A.; Paxton, T.; Dell, A.; Rogers, M.; Sinden, R. E.; Morris, H. R.; Nature 1998, 392, 289.

19. Roma, G.; Braccio, M. D.; Grossi, G.; Mattioli, F.; Ghia, M.; Eur. J. Med. Chem. 2000, 35, 1021.

20. Chen, Y. L.; Fang, K. C.; Sheu, J. Y.; Hsu, S. L.; Tzeng, C. C.; J. Med. Chem. 2001, 44, 2374.

21. Kalluraya, B.; Sreenivasa, S.; Il Farmaco 1998, 53, 399.

22. Maguire, M. P.; Sheets, K. R.; McVety, K.; Spada, A. P.; Zilberstein, A.; J. Med. Chem. 1994, 37, 2129.

23. Huma, H. Z. S.; Halder, R.; Kalra, S. S.; Das, J.; Iqbal, J.; Tetrahedron Lett. 2002, 43, 6485.

24. Dube, D.; Blouin, M.; Brideau, C.; Chan, C. C.; Desmarais, S.; Ethier, D.; Falgueyret, J. P.; Friesen, R. W.; Girard, M.; Girard Y.; Guay, J.; Riendeau, D.; Tagari, P.; Young, R. N.; Bioorg. Med. Chem. Lett. 1998, 8, 1255.
25. Fournet, A.; Hocquemiller, R.; Roblot, F.; Cavé, A.; Richomme, P.; Bruneton, J.; J. Nat. Prod. 1993, 56, 1547.

26. Franck, X.; Fourner, A.; Prina, E.; Mahieux, R.; Hocquemiller, R.; Figadère, B.; Bioorg. Med. Chem. Lett. 2004, 14, 3635.

27. Fournet, A.; Ferreira, M. E.; Rojas de Arias, A.; Torres de Ortiz, S.; Fuentes, S.; Nakayama, H.; Schinini, A.; Hocquemiller, R.; Antimicrob. Agents Chemother. 1996, 40, 2447.

28. Du, W.; Curran, D. P.; Org. Lett. 2003, 5, 1765.

29. Lindsay, D. M.; Dohle, W.; Jensen, A. E.; Kopp, F.; Knochel, P.; Org. Lett. 2002, 4, 1819.

30. Dormer, P. G.; Eng, K. K.; Farr, R. N.; Humphrey, G. R.; McWilliams, J. C.; Reider, P. J.; Sager, J. W.; Volante, R. P.; J. Org. Chem. 2003, 68, 467.

31. Ghorbani-Vaghei, R.; Akbari-Dadamahaleh, S.; Tetrahedron Lett. 2009, 50, 1055.

32. Wang, G. W.; Jia, C. S.; Dong, Y. W.; Tetrahedron Lett. 2006, 47, 1059 .

33. Zolfigol, M. A.; Salehi, P.; Ghaderi, A.; Shiri, M.; Tanbakouchian, Z.; J. Mol. Catal. A: Chem. 2006, 259, 253.

34. Lavrado, J.; Paulo, A.; Gut, J.; Rosenthal, P. J.; Moreira, R.; Bioorg. Med. Chem. Lett. 2008, 18, 1378.

35. Chen, Y. L.; Hung, H. M.; Lu, C. M.; Li, K. C.; Tzeng, C. C.; Bioorg. Med. Chem. 2004, 12, 6539.

36. Yang, D. Q.; Jiang, K. L.; Li, J. N.; Xu, F.; Tetrahedron 2007, 63, 7654 .

37. Zora, M.; Veliogluğlu, Ö.; J. Organomet. Chem. 2008, 693, 2159.

38. Majumdar, K. C.; Taher, A.; Debnath, P.; Synthesis 2009, 793.

39. Yu, X. Y.; Hill, J. M.; Yu, G.; Yang, Y. F.; Kluge, A. F.; Keith, D.; Finn, J.; Gallant, P.; Silverman, J.; Lim, A.; Bioorg. Med. Chem. Lett. 2001, 11, 541.

40. Meléndez Gómez, C. M.; Kouznetsov, V. V.; Sortino, M. A.; Alvarez, S.; Zacchino, S. A.; Bioorg. Med. Chem. 2008, 16, 7908.

41. Wu, L. H.; Yang, D. Q.; Chin. J. Org. Chem. 2009, 7, 1122.

42. Gao, W. T.; Zhang, C. H.; Li, Y.; Jiang, Y.; Chin. J. Org. Chem. 2009, 29, 1423.

43. Li, Y.; Zhang, C. H.; Sun, M. C.; Gao, W. T.; J. Heterocycl. Chem. 2009, 46, 1190.

44. Srivastava, V.; Negi, A. S.; Kumar, J. K.; Faridi, U.; Sisodia, B. S.; Daroka, M. P.; Luqman, S.; Khanuja, S. P. S.; Bioorg. Med. Chem. Lett. 2006, 16, 911.

45. Goel, A.; Dixit, M.; Tetrahedron Lett. 2004, 45, 8819.

46. Piloto, A. M.; Costa, S. P. G.; Goncalves, M. S. T.; Tetrahedron Lett. 2005, 46, 4757.

47. Srivastava, V.; Negi, A. S.; Kumar, J. K.; Faridi, U.; Sisodia, B. S.; Darokar, M. P.; Luqman, S.; Khanuja, S. P. S.; Bioorg. Med. Chem. Lett. 2006, 16, 911.

Received: October 19, 2009 Web Release Date: February 4, 2010 


\section{A Novel One-pot Three-step Synthesis of 2-(1-Benzofuran-2-yl)quinoline-3- carboxylic Acid Derivatives}

\section{Wentao Gao, * Chaohua Zhang and Yang Li}

Institute of Superfine Chemicals, Bohai University, Keji Street, Jinzhou, 121000, P. R. China

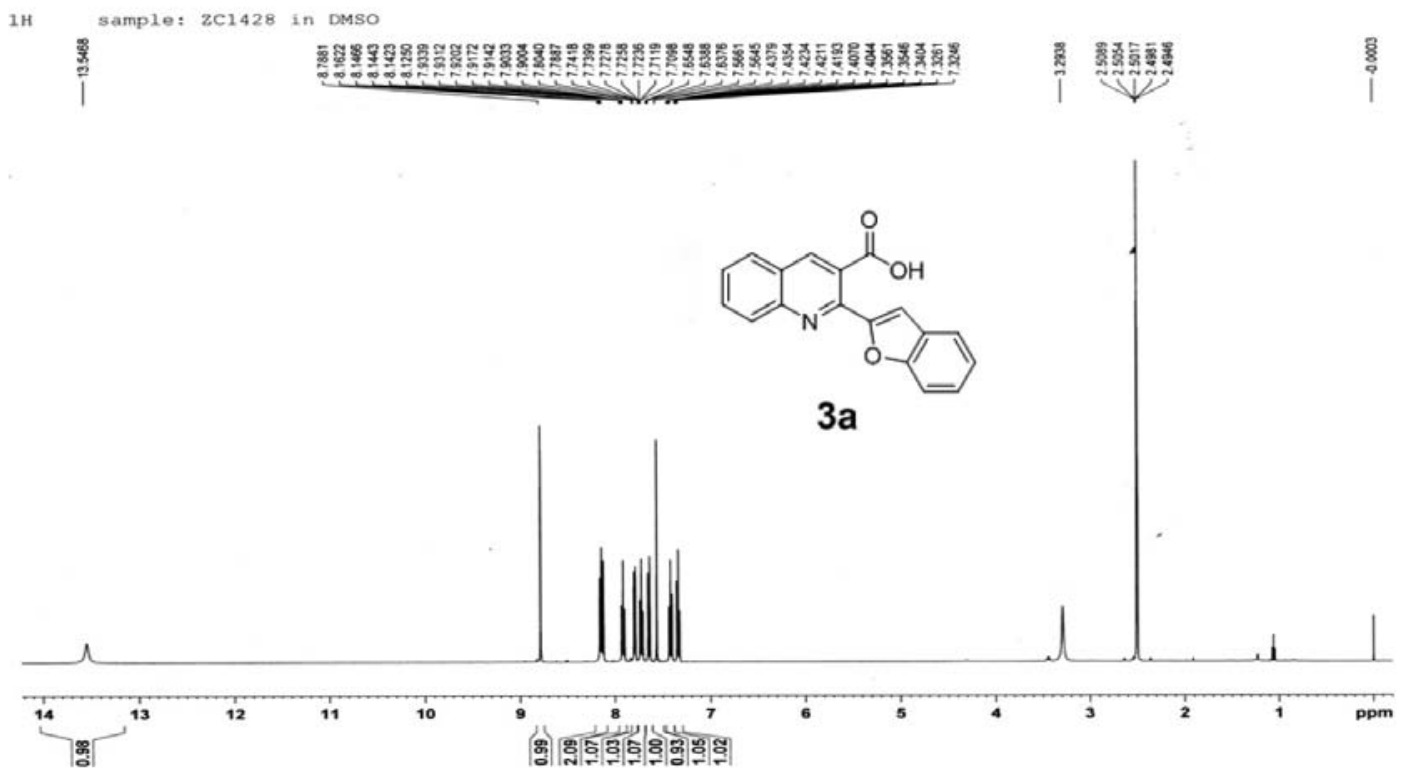

Figure S1. ${ }^{1} \mathrm{H}$ NMR spectrum of $\mathbf{3 a}$. 


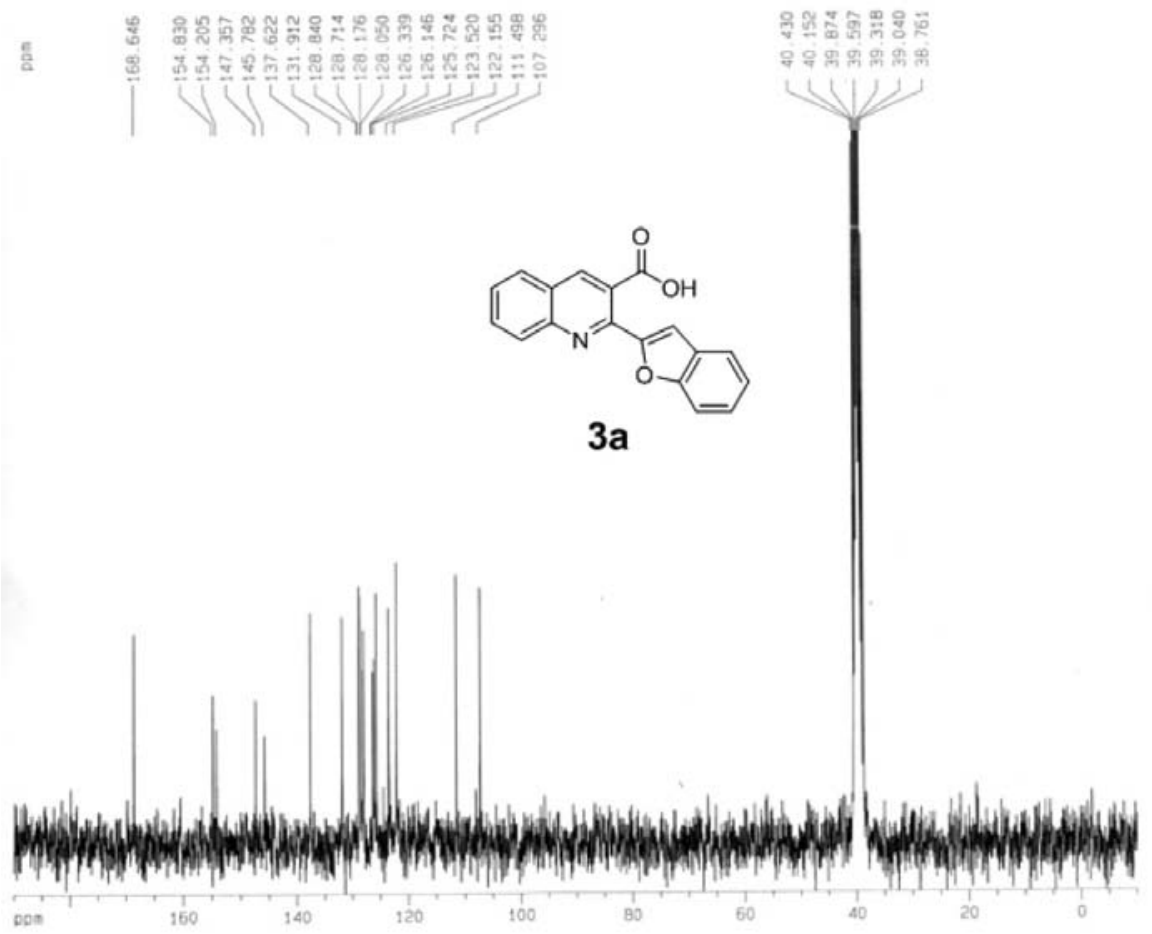

Figure S2. ${ }^{13} \mathrm{C}$ NMR spectrum of $\mathbf{3 a}$.

$1 \mathrm{H}$
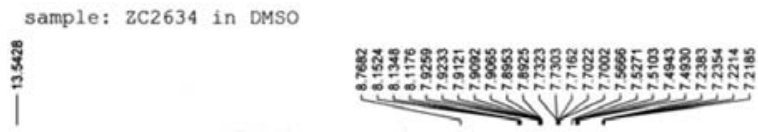

3b
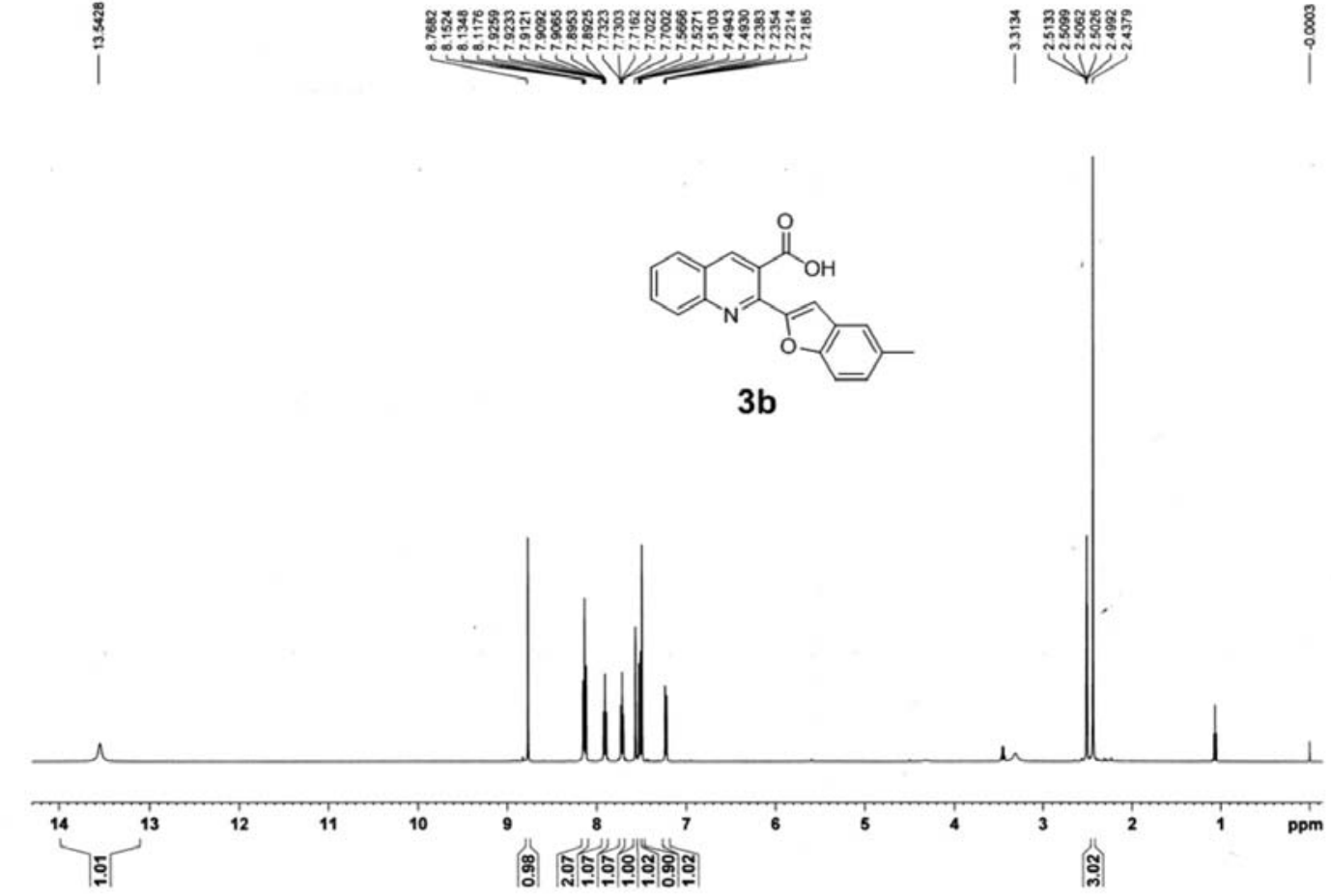

i

Figure S3. ${ }^{1} \mathrm{H}$ NMR spectrum of $\mathbf{3 b}$. 
Vol. 21, No. 5, 2010

Gao et al.

SB

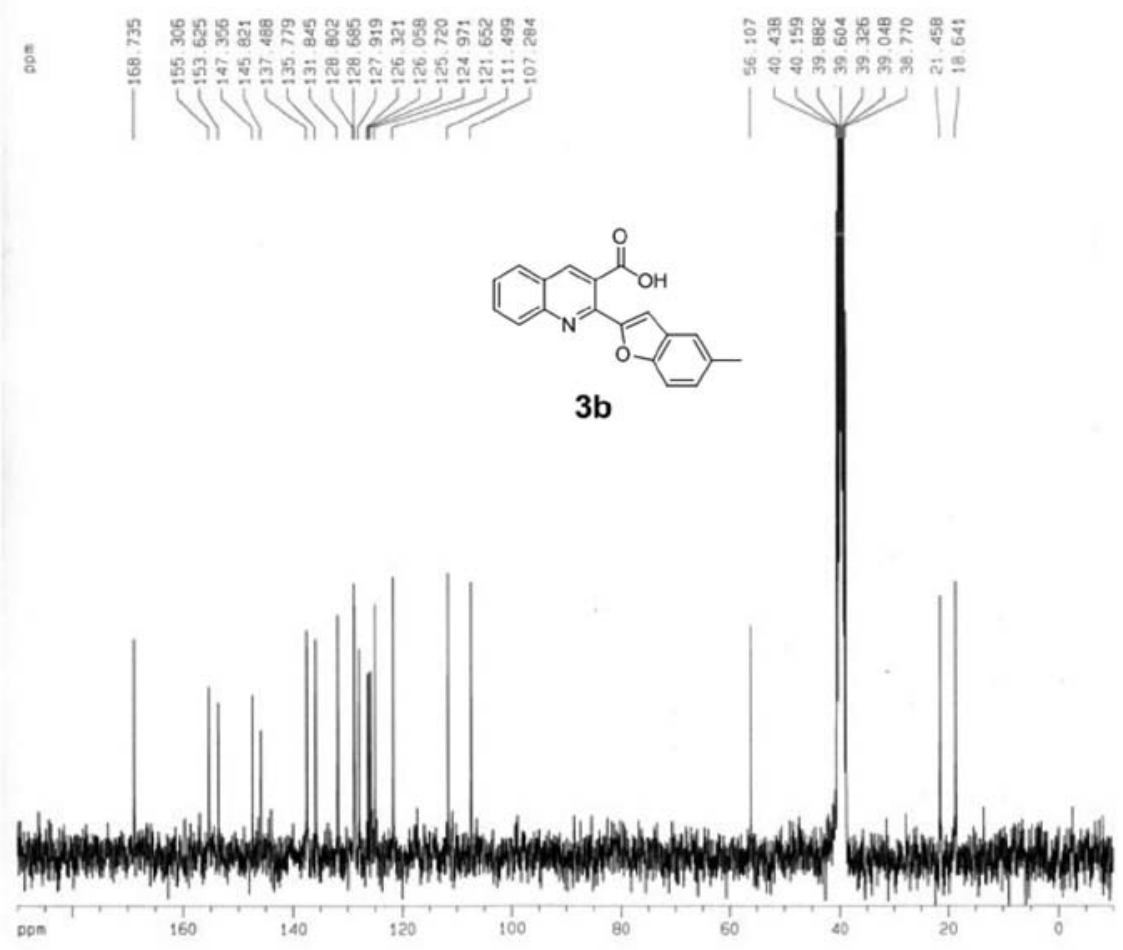

Figure S4. ${ }^{13} \mathrm{C}$ NMR spectrum of $\mathbf{3 b}$.
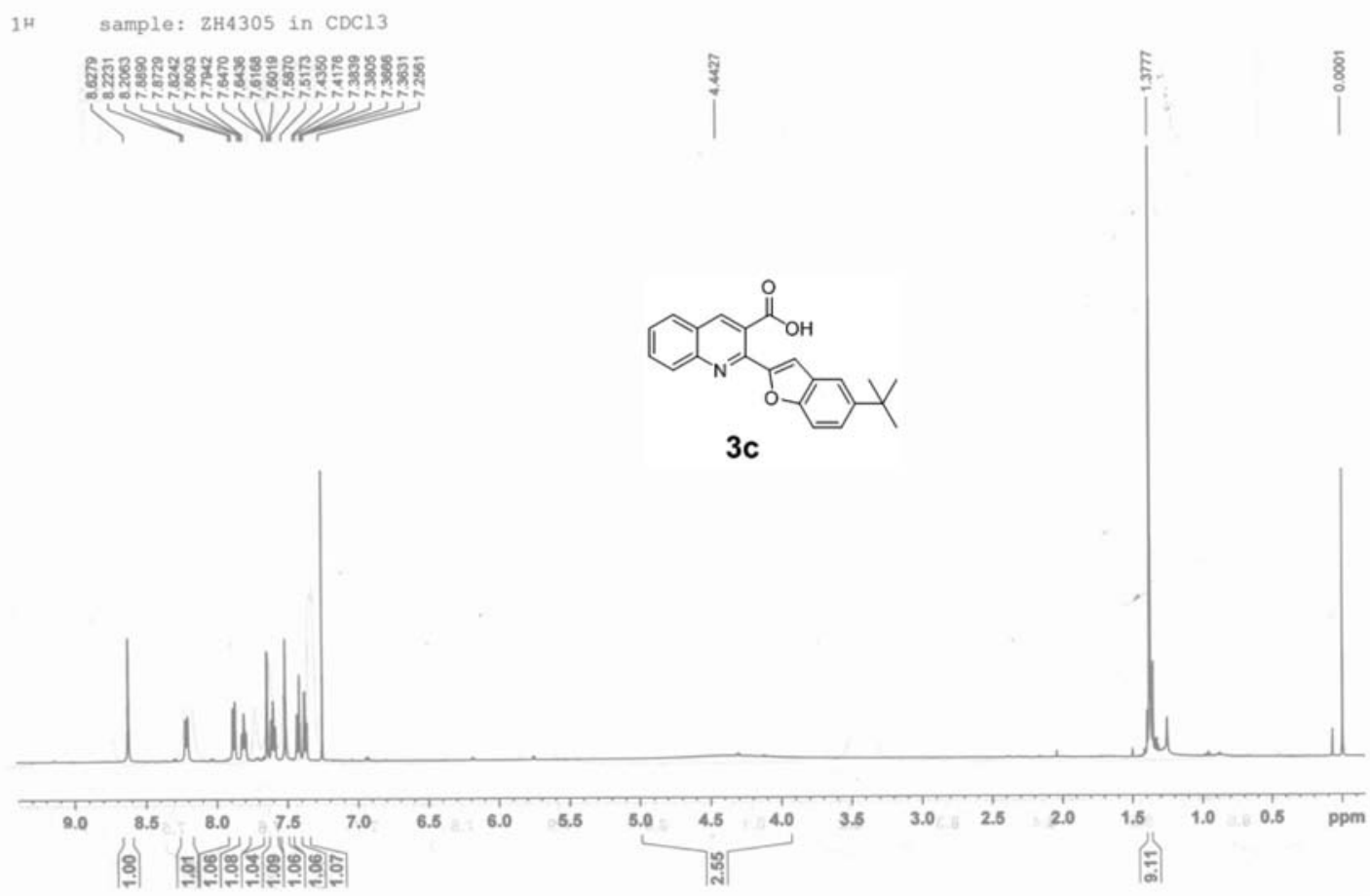

Figure S5. ${ }^{1} \mathrm{H}$ NMR spectrum of $\mathbf{3 c}$. 

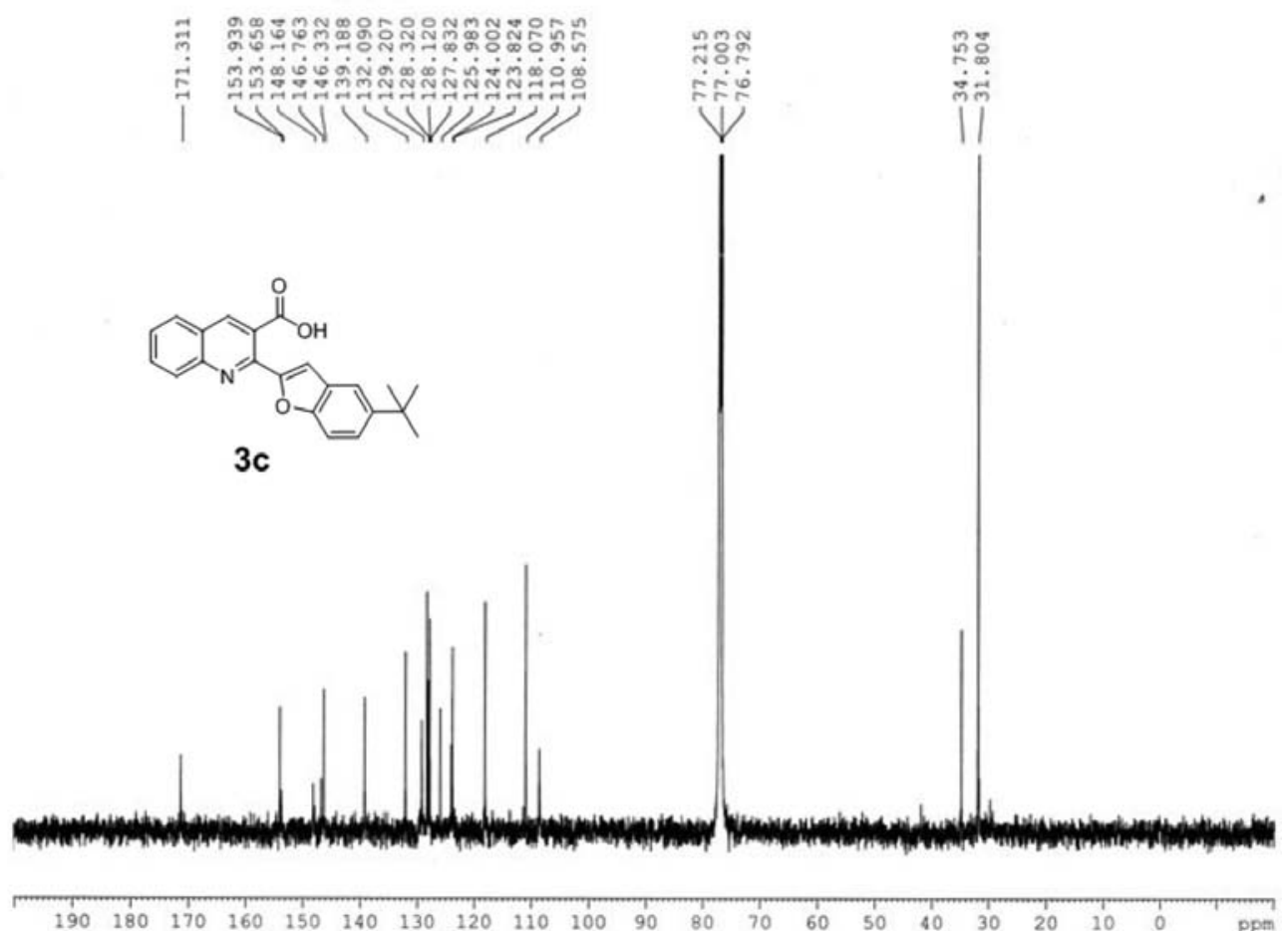

Figure S6. ${ }^{13} \mathrm{C}$ NMR spectrum of $\mathbf{3 c}$.

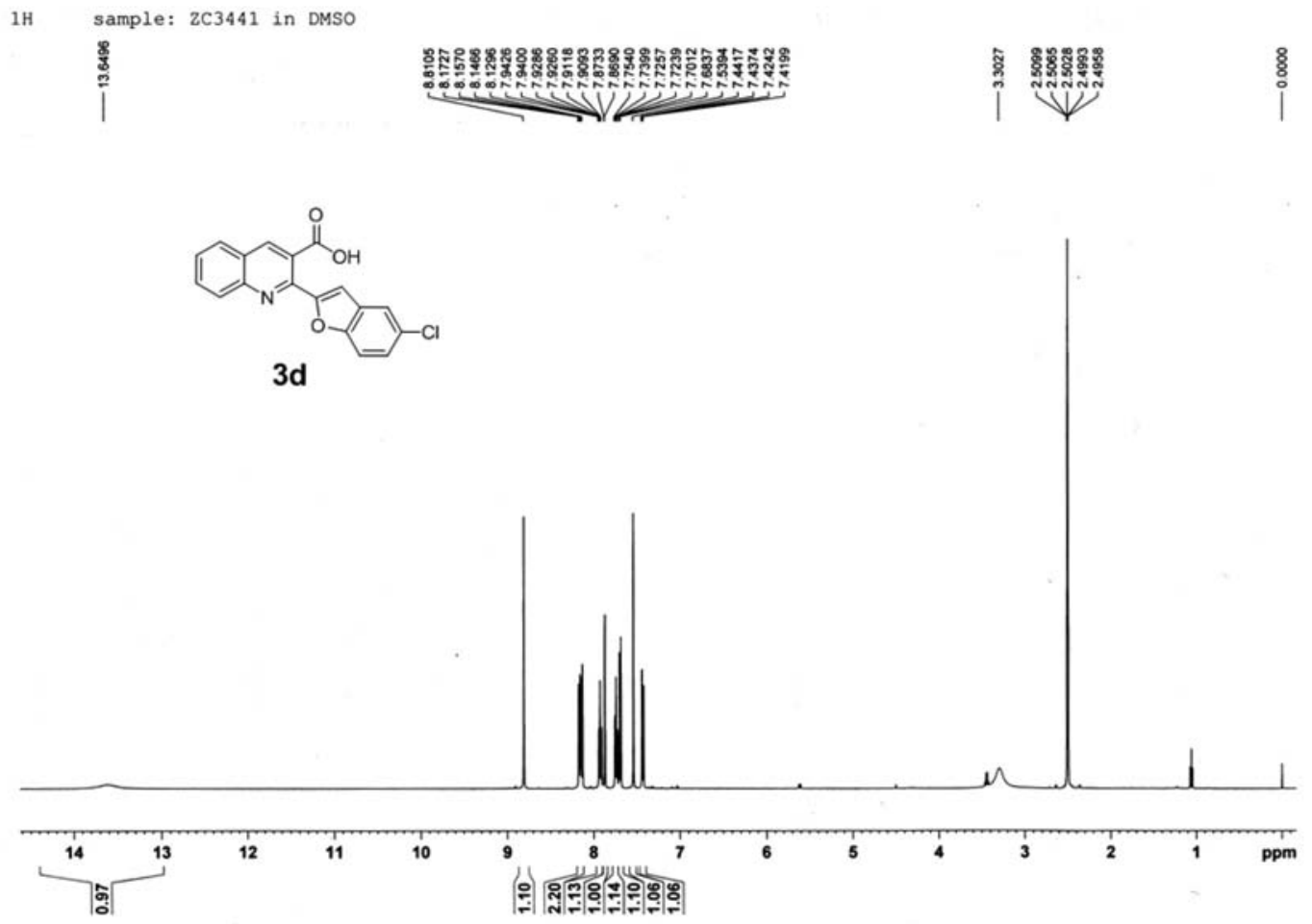

Figure S7. ${ }^{1} \mathrm{H}$ NMR spectrum of $\mathbf{3 d}$. 


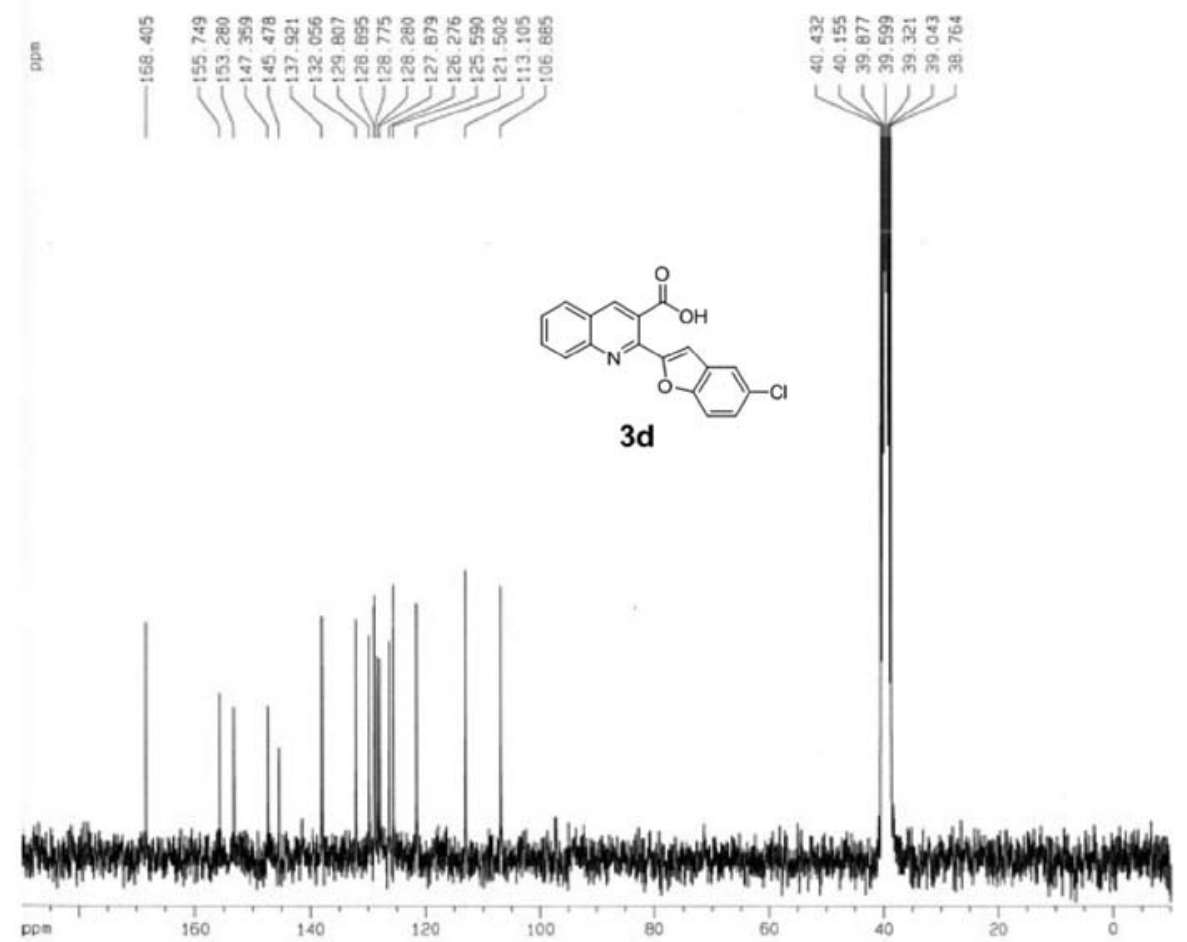

Figure S8. ${ }^{13} \mathrm{C}$ NMR spectrum of $\mathbf{3 d}$.
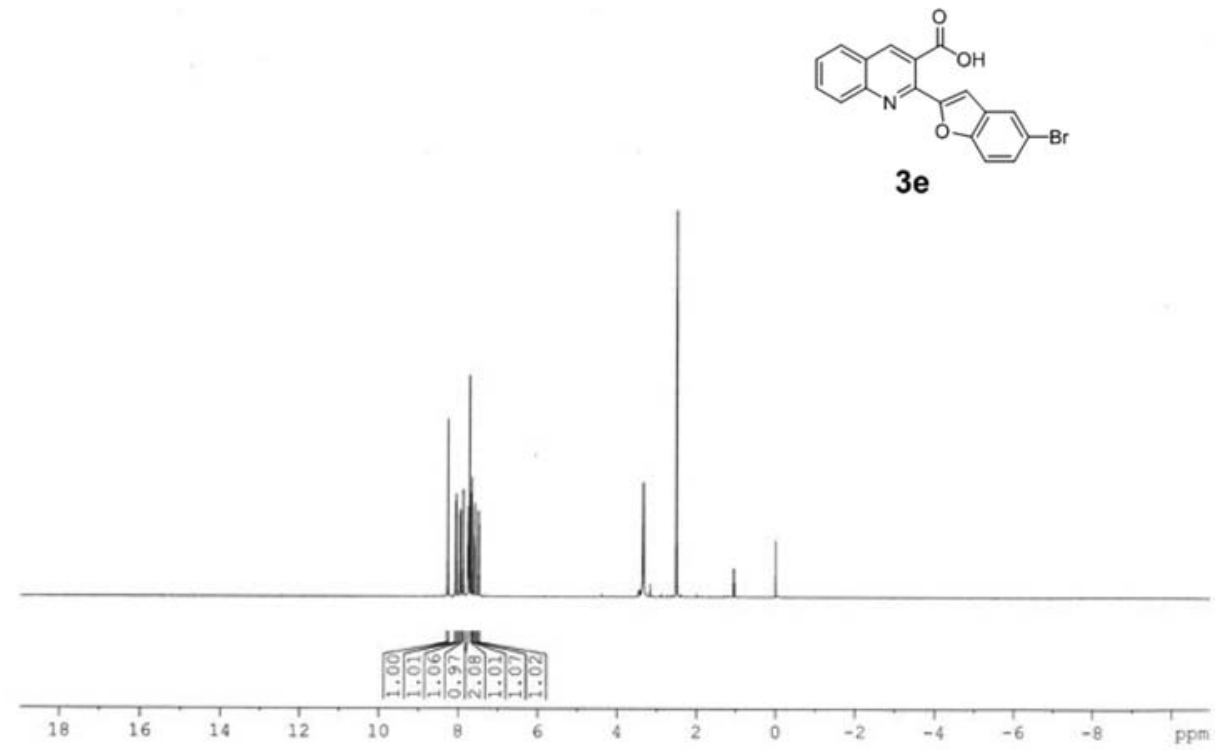

Figure S9. ${ }^{1} \mathrm{H}$ NMR spectrum of $\mathbf{3 e}$ 

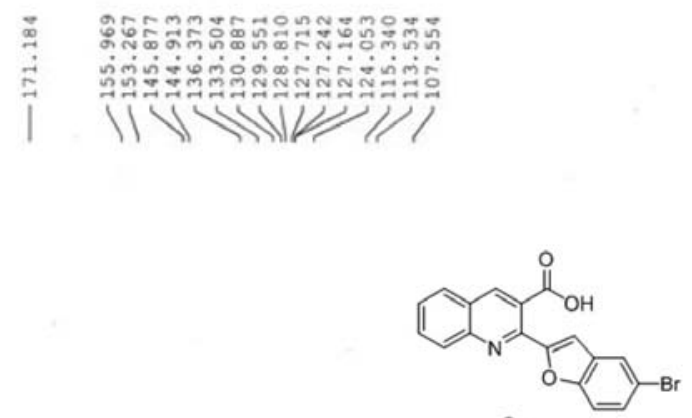

$3 e$

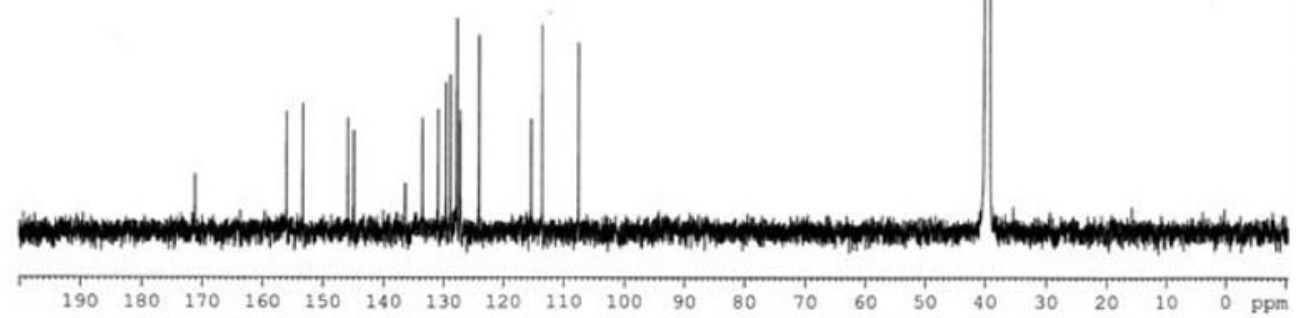

Figure S10. ${ }^{13} \mathrm{C}$ NMR spectrum of $\mathbf{3 e}$.

$1 \mathrm{H}$
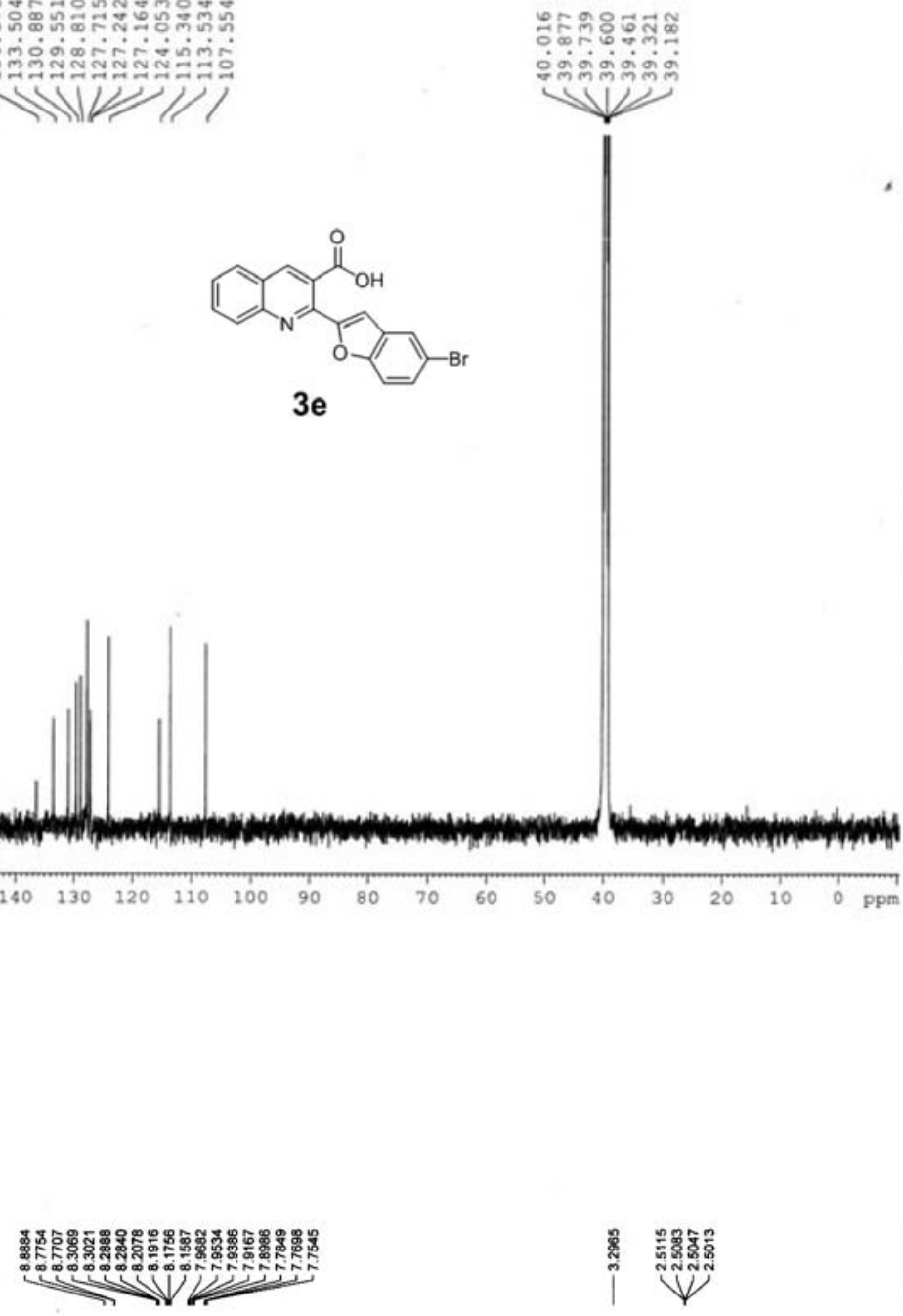
sample: 200927 in DMSO 要<smiles>O=C(O)c1cc2ccccc2nc1-c1cc2cc([N+](=O)[O-])ccc2o1</smiles>

$3 \mathbf{f}$

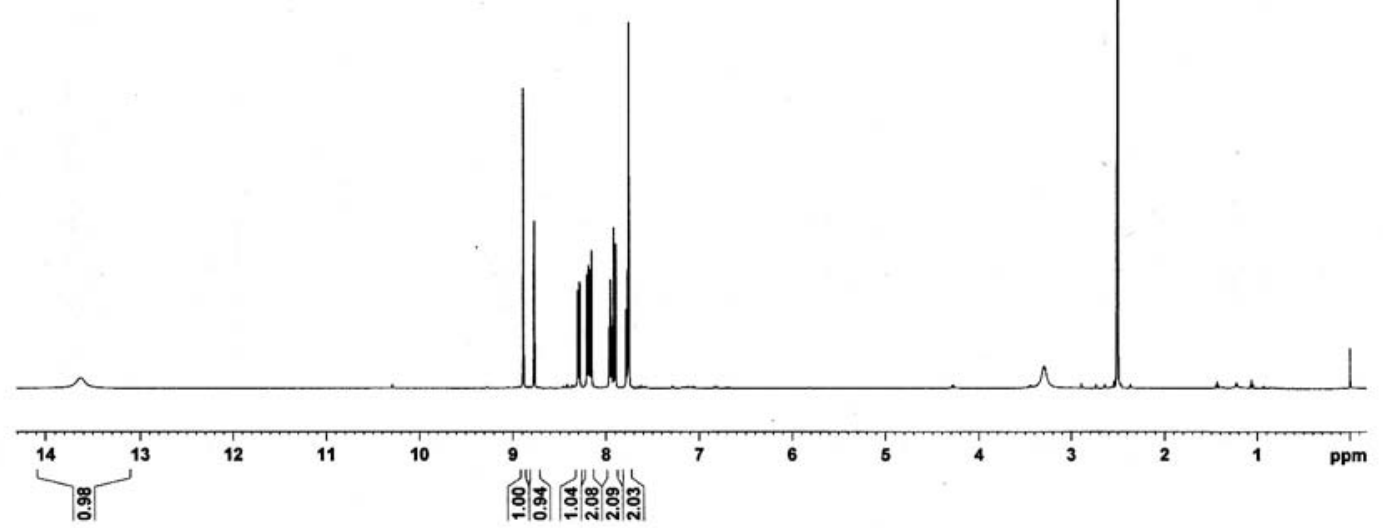

Figure S11. ${ }^{1} \mathrm{H}$ NMR spectrum of $\mathbf{3 f}$. 


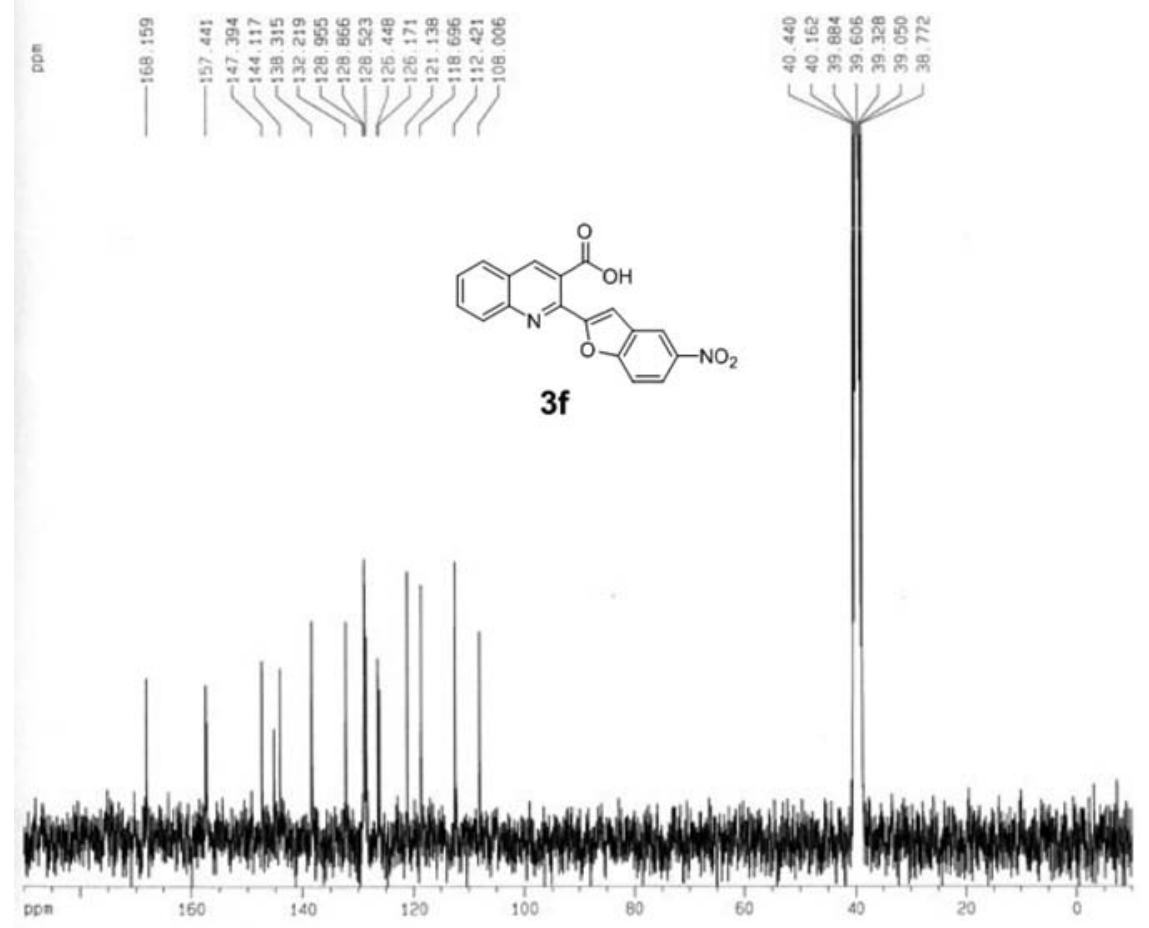

Figure S12. ${ }^{13} \mathrm{C}$ NMR spectrum of $\mathbf{3 f}$.

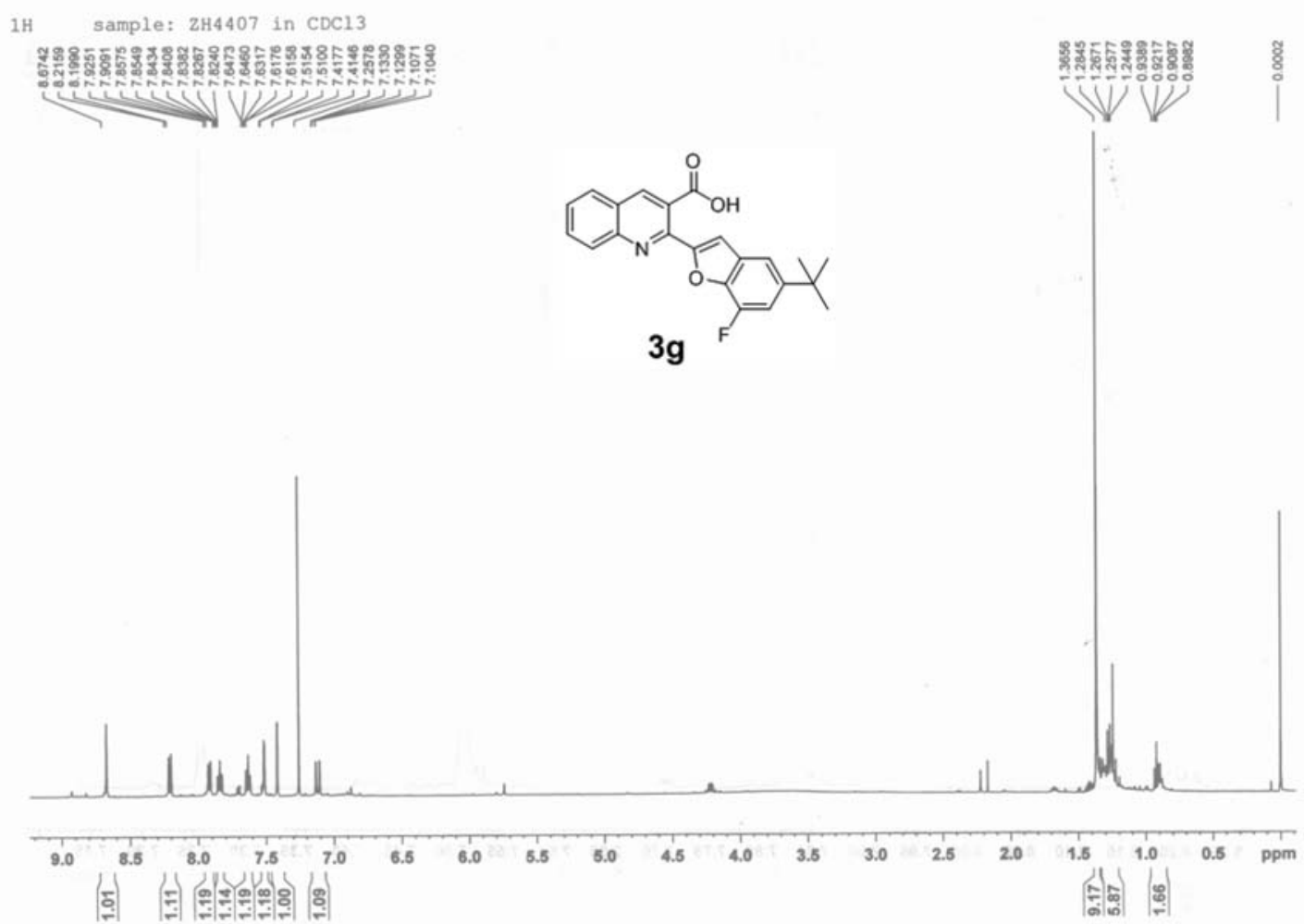

Figure S13. ${ }^{1} \mathrm{H}$ NMR spectrum of $\mathbf{3 g}$. 

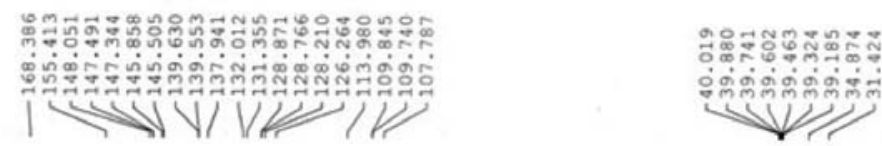

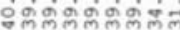

$+$
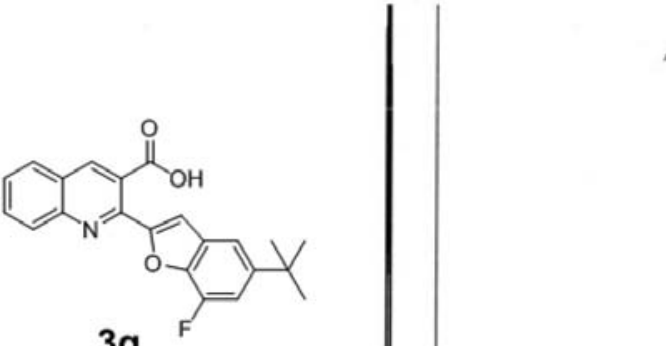

$3 g$

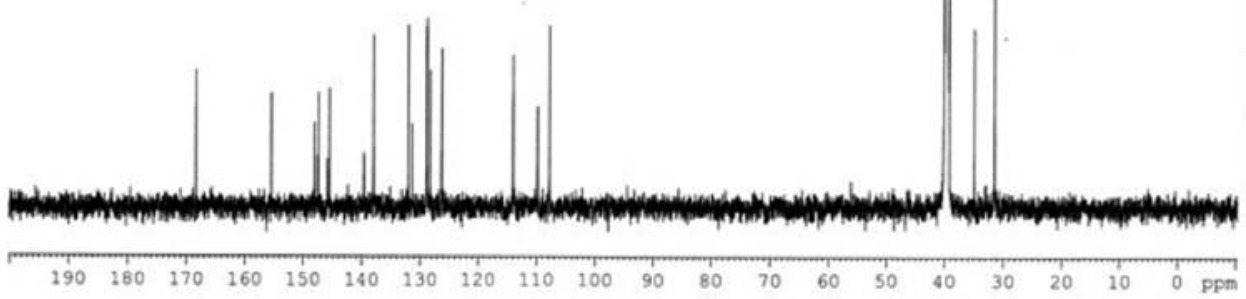

Figure S14. ${ }^{13} \mathrm{C}$ NMR spectrum of $\mathbf{3 g}$.

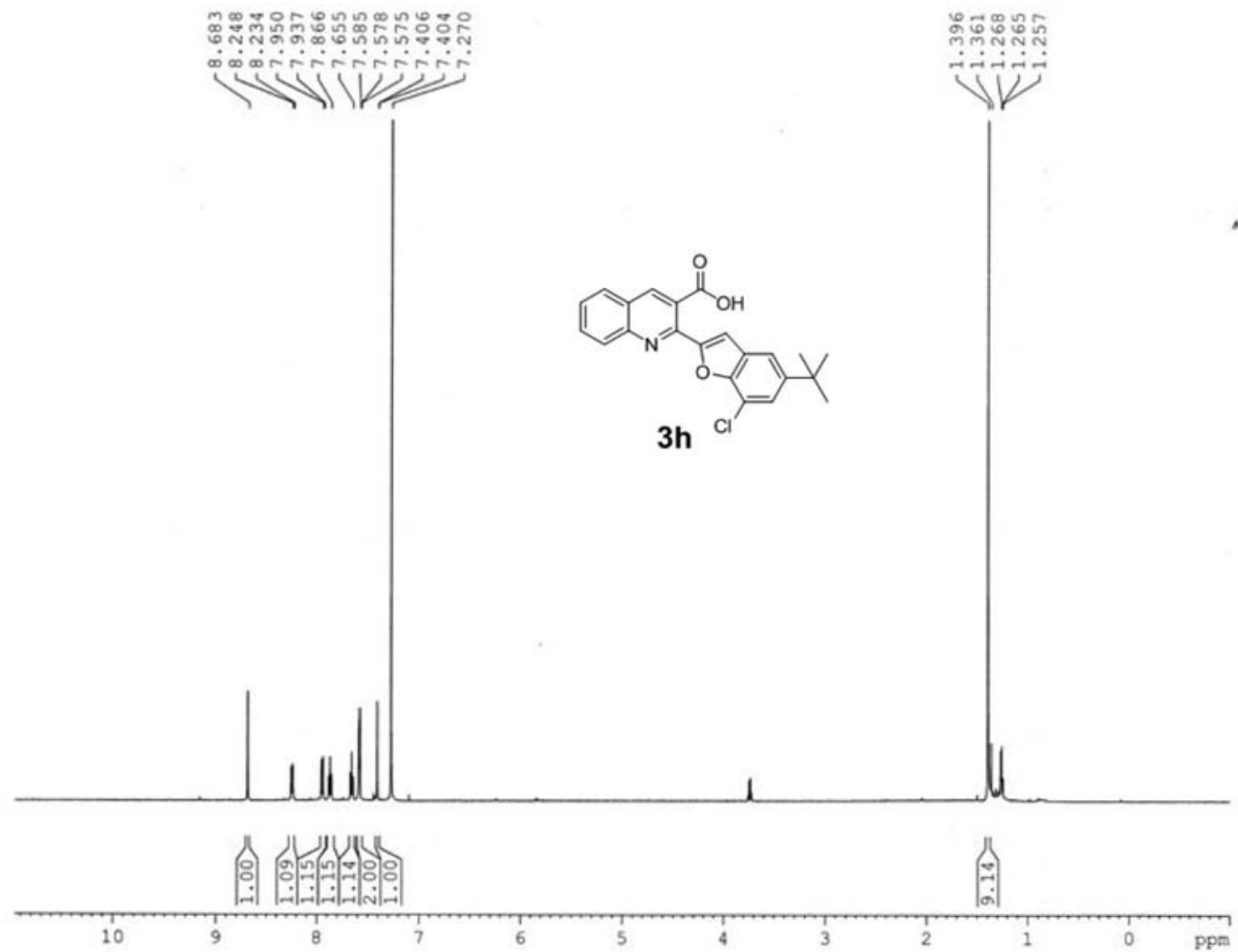

Figure S15. ${ }^{1} \mathrm{H}$ NMR spectrum of $\mathbf{3 h}$. 

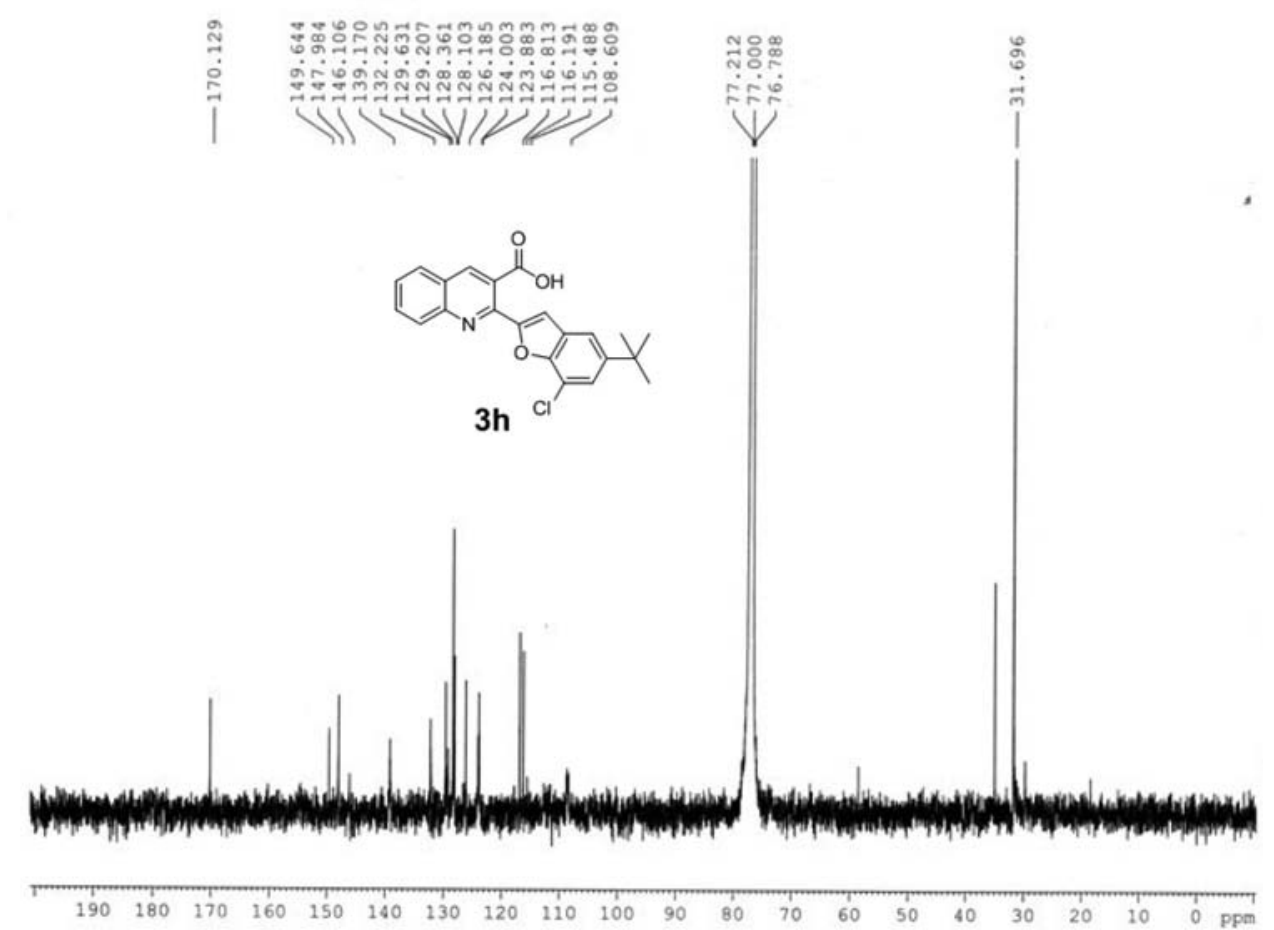

Figure S16. ${ }^{13} \mathrm{C}$ NMR spectrum of $\mathbf{3 h}$.

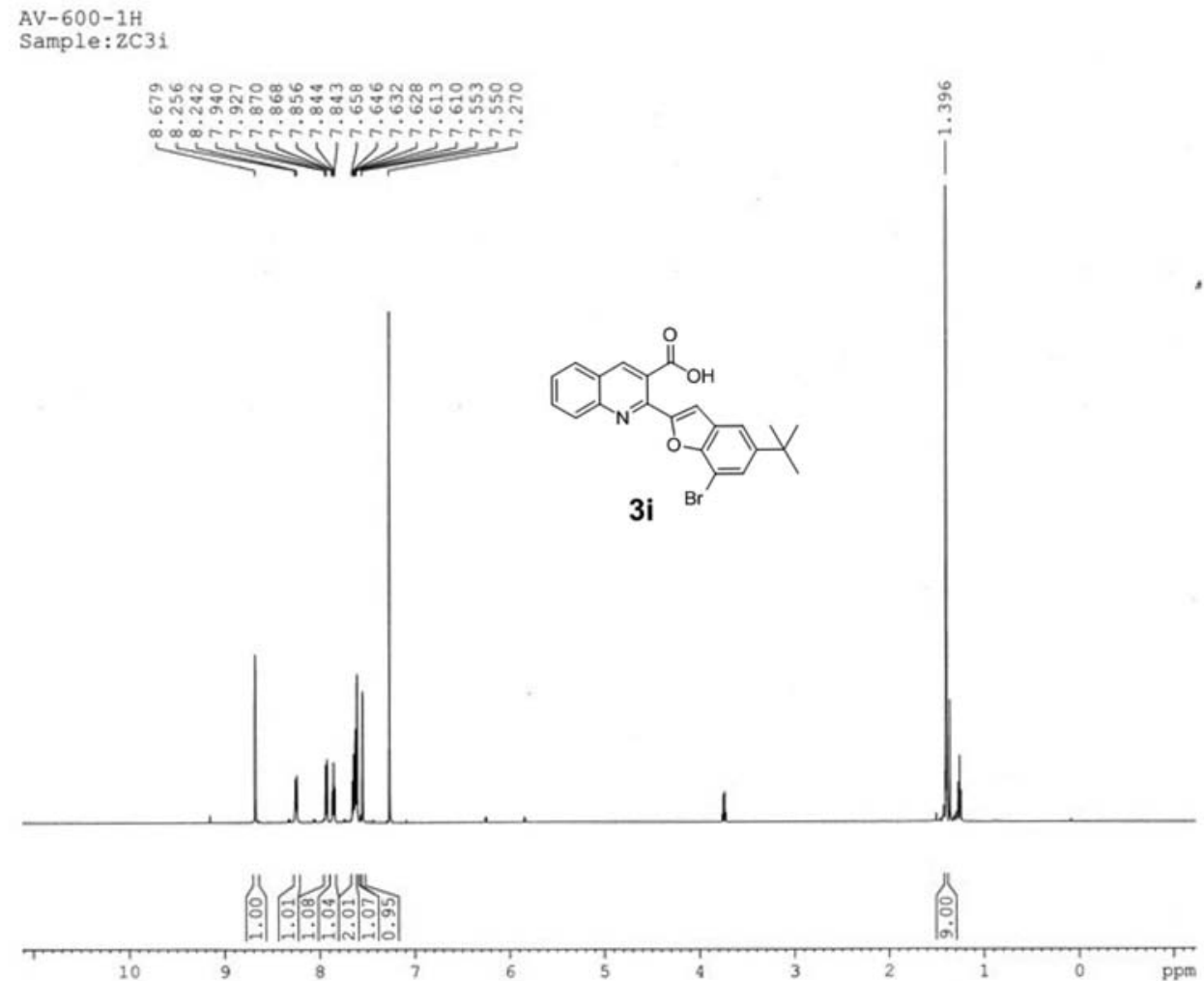

Figure S17. ${ }^{1} \mathrm{H}$ NMR spectrum of $\mathbf{3 i}$. 

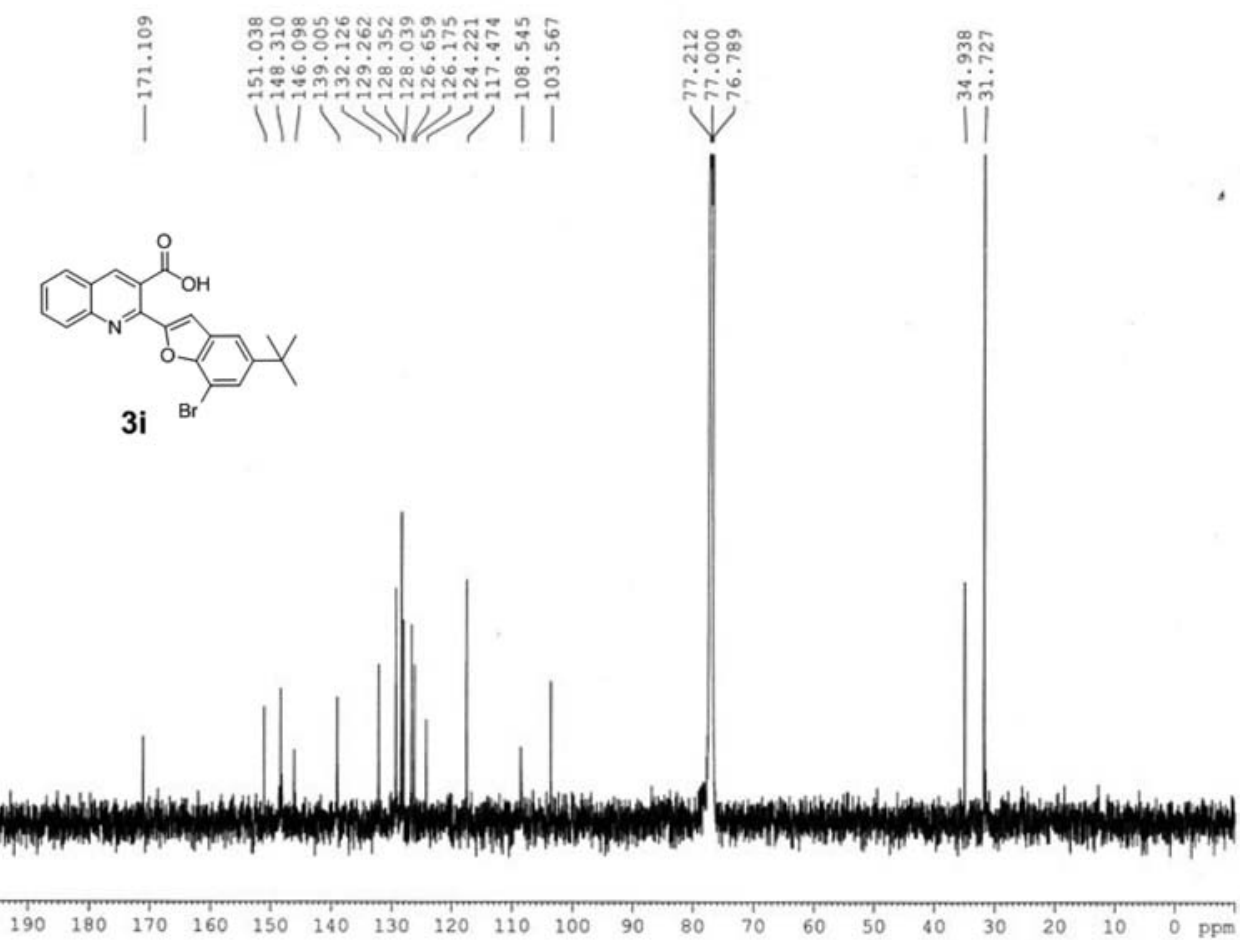

Figure S18. ${ }^{13} \mathrm{C}$ NMR spectrum of $\mathbf{3 i}$.

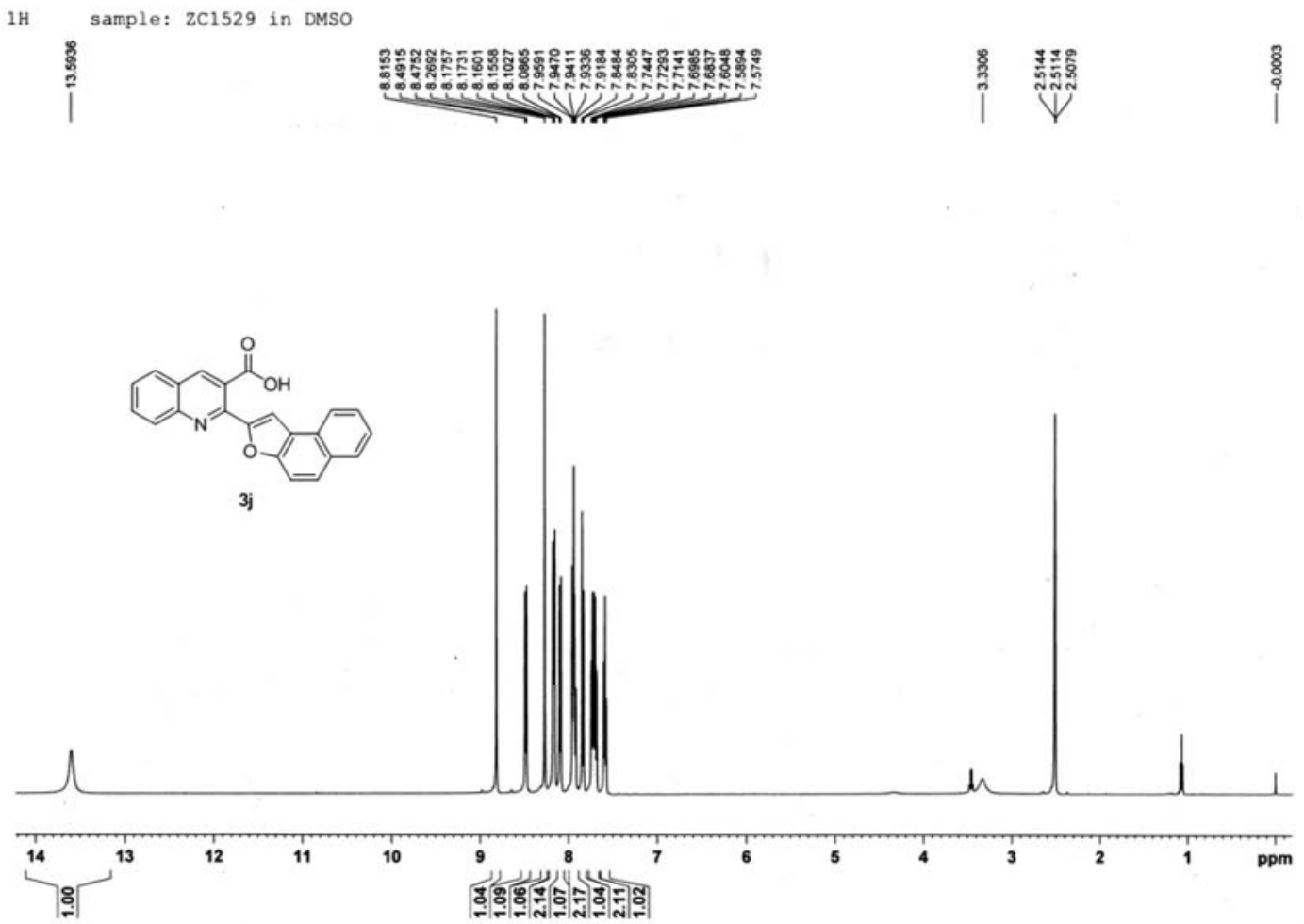

Figure S19. ${ }^{1} \mathrm{H}$ NMR spectrum of $\mathbf{3 j}$. 


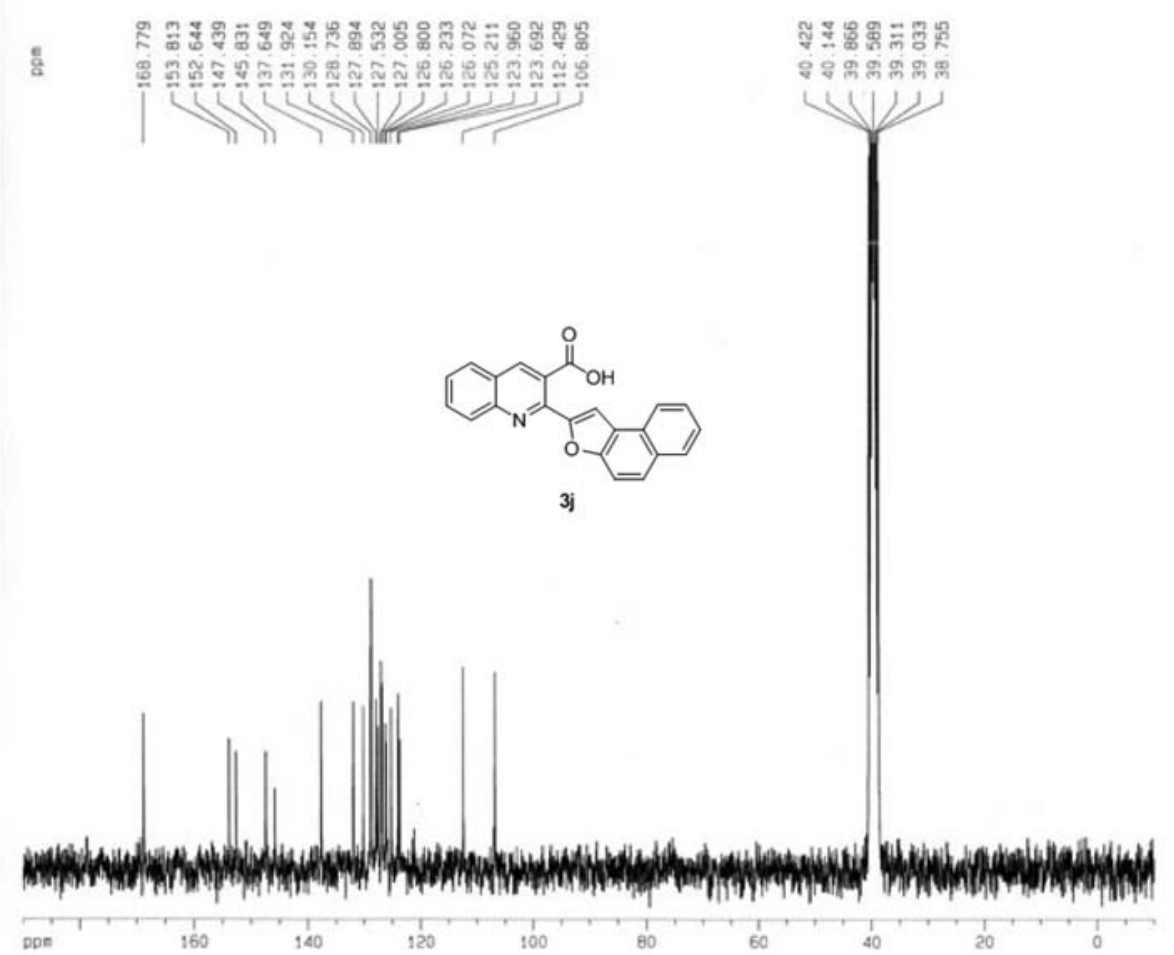

Figure S20. ${ }^{13} \mathrm{C}$ NMR spectrum of $\mathbf{3 j}$.

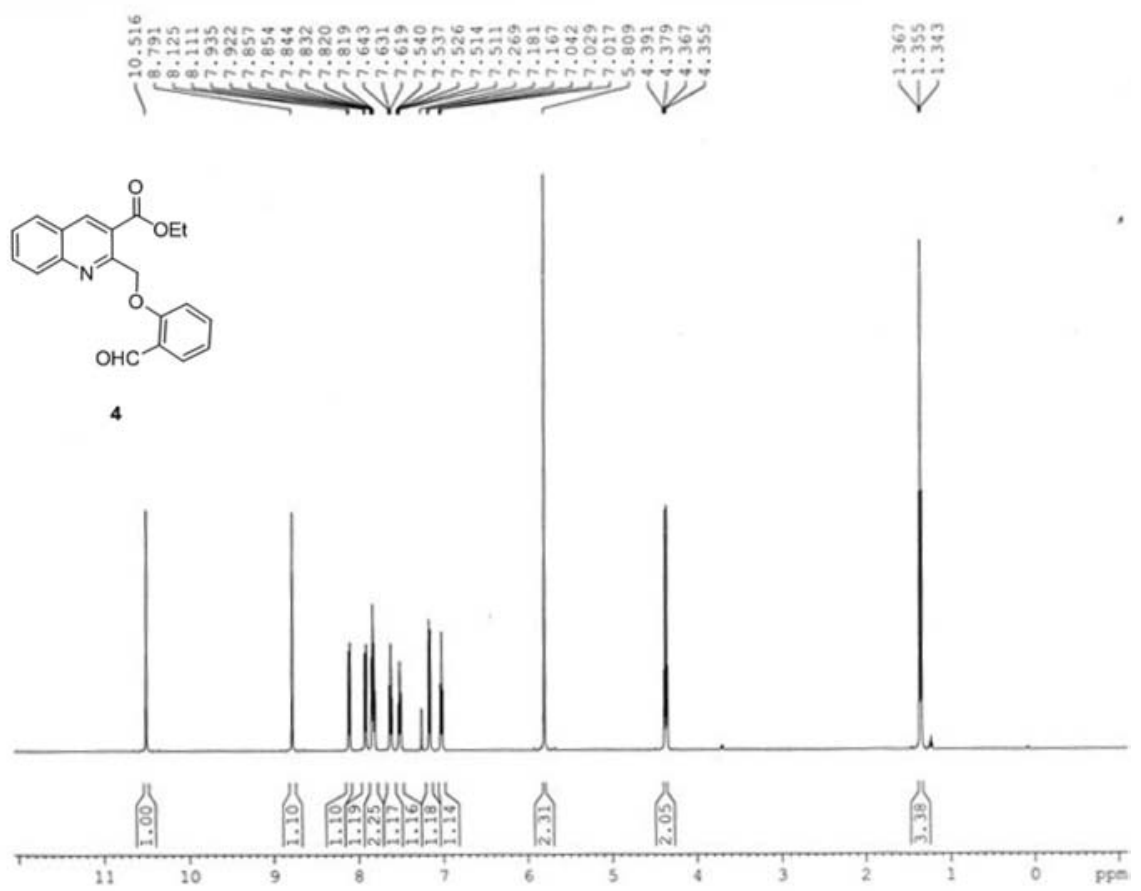

Figure S21. ${ }^{1} \mathrm{H}$ NMR spectrum of 4. 


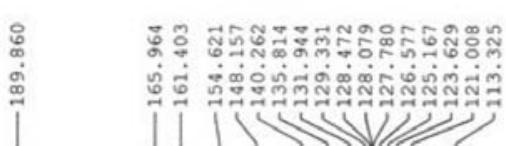

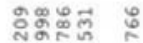

संखिं की

สำ

ธี่ำกี

VI

$\stackrel{i}{i}$<smiles>CCOc1ccccc1OC=O</smiles>

4
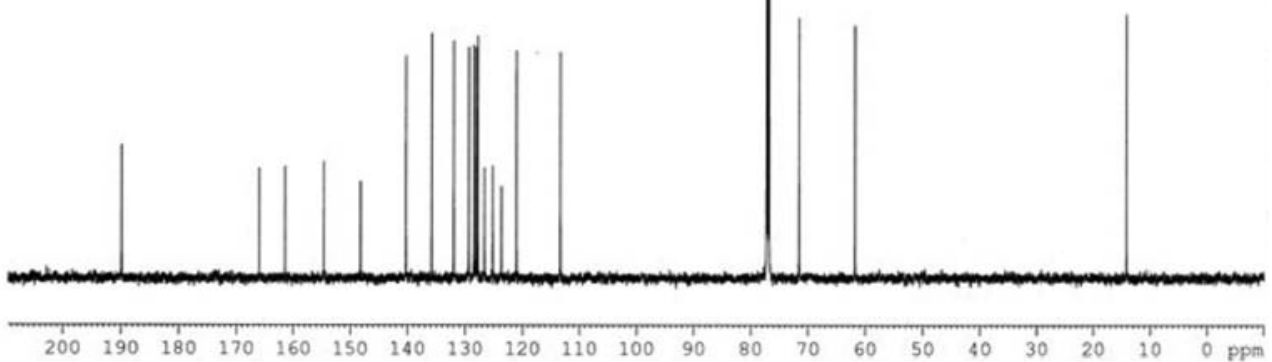

Figure S22. ${ }^{13} \mathrm{C}$ NMR spectrum of 4. 